\title{
Canonical coordinates and Bergmann metrics
}

\author{
WEI-DONG RUAN ${ }^{1}$
}

In this paper we will discuss local coordinates canonically corresponding to a Kähler metric. We will also discuss the $C^{\infty}$ convergence of Bergmann metrics following Tian's result on $C^{2}$ convergence of Bergmann metrics. At the end we present an interesting characterization of ample line bundle that could be useful in arithmetic geometry.

\section{Contents.}

1 Introduction and Background . . . . . . . . . . . . 589

2 Construction of K-coordinates . . . . . . . . . . . . . . 591

3 Tian's peak sections and Bergmann metrics . . . . . . . 596

4 Combinatorics Involved . . . . . . . . . . . . . . 603

5 Combinatoric Approach . . . . . . . . . . . . . . 6 610

6 Geometric Approach . . . . . . . . . . . . . . . . . 6623

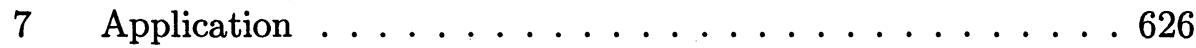

\section{Introduction and Background.}

For any real analytic Kähler manifold, there exists a canonically defined local coordinate in a neighborhood of any point, which depends antiholomorphically on the point. Since the coordinates canonically correspond to Kähler metrics, we will call them K-coordinates, representing Kähler coordinates. These coordinates first come to our attention from the literature in mirror symmetry. It is given a nice geometric interpretation by Kontsevich. We will discuss this interpretation and see its geometric implications.

$\mathrm{K}$-coordinates actually were already quite well known in early mathematics literature. To author's knowledge, they first appeared in [B]. Given the "canonical" nature of these coordinates, it is quite surprising to me that

\footnotetext{
${ }^{1}$ Partially supported by Sonderforschungsbereich 237 .
} 
they were not widely used in the literature, although a finite order approximation of it was used in Tian's proof of $C^{2}$ convergence of Bergmann metrics $[\mathrm{T}]$. It is very interesting, at least to the author, to see how useful these coordinates are in handling problems in Kähler geometry and other fields. We will illustrate their use through one example: the proof of $C^{\infty}$ convergence of Bergmann metrics.

It was first conjectured by Yau that Bergmann metrics induced by a Kähler-Einstein metric should converge to the original Kähler-Einstein metric. Later, Tian proved that Bergmann metrics induced by any Kähler metric of integral Kähler class will converge to that Kähler metric in $C^{2}$ norm. The problem is roughly as follows. For $(M, g)$ a Kähler manifold with the Kähler class $\omega_{g} \in H^{2}(M, \mathbf{Z})$, one can realize $\omega_{g}$ as the first Chern class of an ample line bundle $L$. Multiples $L^{m}$ will induce certain canonical embeddings of $M$ into projective spaces by all its sections for $m$ large.

$$
\varphi_{m}: M \longrightarrow \mathbf{C P}^{\mathbf{N}_{\mathbf{m}}}
$$

Pull backs of Fubini-Study metrics $\varphi_{m}^{*} g_{F S}$ on $M$ are usually called Bergmann metrics. They look like a nice collection of metrics canonically associated to the Kähler manifold, very much like polynomials. It then sounds natural to expect that a suitably chosen sequence of Bergmann metrics will converge to the original Kähler metric. The precise definitions and statements will be given in section 3 .

The starting point for Tian's proof is the construction of a sequence of "peak sections", which, when used correctly, will reduce the problem to certain kind of computation. He then handled these computations by brute force, for which he already ran into quite complicated computation in proving the $C^{2}$ convergence. Higher order convergences do not seem manageable in the same way. Although he believes that the $C^{\infty}$ convergence should be true.

On our way of understanding the K-coordinates, we find Tian's proof very natural in $\mathrm{K}$-coordinate point of view, which enable us to choose a more canonical factor in a lemma to make it simpler. This turns out to be a crucial modification, which makes the resulting computation problem more tackable. For instance, we can give a simple combinatoric proof of the $C^{3}$ convergence without serious computation. (See corollary 5.1 and the remark after that.) At least in theory, it turns the $C^{\infty}$ convergence into a purely combinatoric problem, which, in principle, should be solvable. But it turns out the corresponding combinatoric problem is very complicated to solve, if not impossible. (I have more respect and appreciation to combinatoric problems now than before.) Although we were able to solve it to a great extent, 
even deduced an algorithm to prove convergence to arbitrary order, modulo computations that get more and more complicated. (We checked the first non-trivial combinatoric identity, which implies the $C^{5}$ convergence.) We are still not able to solve it completely by purely combinatoric computation. Another advantage of our approach is that the error terms of our estimates are naturally $O\left(\frac{1}{m}\right)$ instead of $O\left(\frac{1}{\sqrt{m}}\right)$.

Finally, it turns out, there is a very simple and elegant geometric proof of $C^{\infty}$ convergence, which even greatly simplifies the Tian's proof for the $C^{2}$ convergence. It reduces the needed computation to very minimal. But it does not seem to uncover the combinatoric mystery of the problem, which involves quite a lot of magical vanishings, which is quite interesting in its own.

Our paper is organized in the following way. In section 2, we will discuss the construction of K-coordinates, especially Kontsevich's interpretation. In section 3, we will introduce the convergence problem of Bergmann metrics, Tian's result, and carry out some delicate argument to reduce the problem to a combinatoric one. In section 4 , We will demonstrate the combinatorics involved, and set up notation and combinatoric framework. In section 5, we try to sort out the complicated combinatorics to a great extent. While these problems are very interesting and challenging, they are not absolutely necessary, and not very easy to get through, therefore can be skipped without serious lossin understanding our geometric proof. In section 6 , we will present the simple geometric proof. In the last section, we will present some interesting application of our results, give a characterization of ample line bundles which could be useful in arithmatic geometry.

\section{Construction of $\mathrm{K}$-coordinates.}

Before getting into the construction of K-coordinates, let's first review some facts from symplectic geometry.

Proposition 2.1. Given a symplectic manifold $(M, \omega)$ with a Lagrangian foliation $\pi: M \longrightarrow Q$, where each fiber of $\pi$ is a Lagrangian submanifold. On the tangent bundle of each Lagrangian submanifold, one can canonically define a flat connection by:

$$
\nabla_{u} v=i_{\omega}^{-1} \mathcal{L}_{u} i_{\omega} v
$$

where $u, v$ are vector fields along the leaves of Lagrangian foliation, and $i_{\omega}: T_{M} \rightarrow T_{M}^{*}$ is defined as $i_{\omega} v=i(v) \omega$. 
Proof. Assume $w$ is a vector field along Lagrangian foliation, we need to check $\left\langle\mathcal{L}_{u} i_{\omega} v, w\right\rangle=0$, and " $\nabla$ " as defined above is a connection.

$$
\left\langle\mathcal{L}_{u} i_{\omega} v, w\right\rangle=u(\omega(v, w))-\omega(v,[u, w])=0
$$

and

$$
\begin{aligned}
\mathcal{L}_{u} i_{\omega}(a v) & =u(a) i_{\omega} v+a \mathcal{L}_{u} i_{\omega} v \\
\mathcal{L}_{(a u)} i_{\omega} v & =i(a u) d\left(i_{\omega} v\right)+d\left(i(a u)\left(i_{\omega} v\right)\right)=a \mathcal{L}_{u} i_{\omega} v
\end{aligned}
$$

Recall that:

$$
\mathcal{L}_{u} \mathcal{L}_{v} \alpha-\mathcal{L}_{v} \mathcal{L}_{u} \alpha=\mathcal{L}_{[u, v]} \alpha
$$

we get the flatness of the connection, therefore define a canonical affine structure on each Lagrangian fiber.

We can also see this in terms of local coordinates, let $q$ be the coordinates of $Q$, and $(p, q)$ be coordinates of $M$. In these coordinates,

$$
\omega=b_{i j} d p^{i} \wedge d q^{j}+a_{i j} d q^{i} \wedge d q^{j} .
$$

The closeness of $\omega$ implies that $\partial\left(b_{i j}\right) / \partial p^{k}=\partial\left(b_{k j}\right) / \partial p^{i}$, which imply that

$$
\omega=d f^{i} \wedge d q^{i}+a_{i j} d q^{i} \wedge d q^{j}
$$

for some $f^{i}$. (Here $a_{i j}$ actually changed due to $q$ derivatives of $f^{i}$.) Now change the coordinates as $p^{i}=f^{i}$, we get

$$
\omega=d p^{i} \wedge d q^{i}+a_{i j} d q^{i} \wedge d q^{j}
$$

here $a_{i j}$ depend only on $q . \quad p$ so defined, gives us the affine structure on every Lagrangian leaf as induced by the connection. The ambiguities on determining $p$ are exactly affine transformations depending on $q$.

We can further simplify the expression of $\omega$ by noticing that $a_{i j} d q^{i} \wedge d q^{j}$ is closed, so

$$
a_{i j} d q^{i} \wedge d q^{j}=d\left(a_{i} d q^{i}\right)=d a_{i} \wedge d q^{i}
$$

and a transformation that depends on $q$ : $p^{i} \longrightarrow p^{i}+a_{i}(q)$, will reduce $\omega$ to the standard form

$$
\omega=d p^{i} \wedge d q^{i}
$$

Without loss of generality, we may fix the coordinate $q$, then the ambiguities of the coordinate $p$ are translations of following type

$$
p^{i} \longrightarrow p^{i}+\frac{\partial f(q)}{\partial q^{i}}
$$


Here $f(q)$ is a function that only depends on $q$.

Now, we recall the construction by Kontsevich. Given a Kähler manifold $(M, \omega)$, where $\omega$ is real analytic. Denote by $\bar{M}$ the same manifold $M$ endowed with the complex structure conjugate to the original one. The diagonal submanifold $M^{\text {diag }}$ of $M \times \bar{M}$ is totally real. Hence the differential form $\omega$ on $M^{\text {diag }}$ has the analytic continuation to the holomorphic form $\omega^{\mathrm{C}}$ in a neighborhood $U$ of $M^{\text {diag }}$. Thus $U$ is a complex symplectic manifold. It is easy to see that the submanifolds $M \times\{\bar{x}\} \cap U$, where $\bar{x} \in \bar{M}$, are Lagrangian. It means that we have a Lagrangian foliation of $\mathrm{U}$. By our above discussion, leaves of such a foliation carry a natural flat affine structure. Hence this gives the construction of holomorphic affine structures on open subsets of $M$ depending antiholomorphically on points of $M$.

Let $(z, w)$ be local coordinates of $M \times \bar{M}$, then $i: M \rightarrow M^{\text {diag }} \hookrightarrow M \times \bar{M}$ can be written as: $i(z)=(z, \bar{z})$. Assume

$$
\omega=g_{i j}(z, \bar{z}) d z^{i} \wedge d \bar{z}^{j}
$$

then

$$
\omega^{\mathbf{C}}=g_{i j}(z, w) d z^{i} \wedge d w^{j}
$$

For any $x \in M$, let $U_{x}=i^{-1}(M \times\{\bar{x}\} \cap U)$, and $\phi_{x}: U_{x} \longrightarrow \mathbf{C}^{\mathbf{n}}$ is a flat coordinate with respect to $x$, as defined above, such that $\phi_{x}(x)=0$ and tangent map at $x$ is an isometry with respect to standard metric on $\mathbf{C}^{\mathbf{n}}$.

Remark 2.1. Maps $\phi_{x}$ are actually parameterized by elements on the total space of the canonical principle $U(n)$ bundle on $M$ with respect to the Kähler metric.

If we take the coordinates $z=\phi_{x}$, since $z$ is already flat coordinate, we have $g_{i j}(z, 0)=\delta_{i j}$, i.e. in the Taylor expansion of $g_{i j}$, all the $(0, l),(l, 0)$ terms vanish. This is equivalent to saying that in each $U_{x}$ we can get a canonical Kähler potential $K_{x}$, which depend on $x$, such that all the $(0, l),(1, l),(l, 1),(l, 0)$ terms in the Taylor expansion of $K_{x}(z)$ vanish except for $(1,1)$ term which equals to $|z|^{2}$. Locally

$$
K_{x}(z)=|z|^{2}+R_{i \bar{j} k l} z^{i} \bar{z}^{j} z^{k} \bar{z}^{l}+O\left(|z|^{5}\right) .
$$

Interestingly, these are the only coordinates which satisfy above vanishing conditions.

Proposition 2.2. For any $x \in M$ there is unique holomorphic coordinate map $z=\phi_{x}$ up to affine transformation, for which there exist a Kähler 
potential $K_{x}(z)$ on $M$ such that all the $(0, l),(1, l),(l, 1),(l, 0)$ terms in the Taylor expansion of $K_{x}(z)$ vanish except for $(1,1)$ term which equal to $|z|^{2}$. These are exactly the $K$-coordinates.

Remark 2.2. It was in this form that $K$-coordinate first appeared in mathematics literature $[\mathrm{B}]$ and recently rediscovered in physics literature.

In above arguments, we did not worry about convergence of various power series, which in principle could cause problems. Now we would like to make some comments to address these problems.

Firstly, if the Kähler metric is actually real analytic or weakly real analytic (as defined later), which is usually true for many Kähler metrics that come up naturally, then those power series will converge in a neighborhood. In general, at least in principle, those power series may not converge. But in most cases, we only need an approximate version of the canonical coordinates, namely coordinates that is "canonical" up to certain order, for which convergence is not a problem. Let the order goes to infinity, we will get a sequence of more and more accurate approximate "canonical" coordinates. These sequences of coordinates usually can do the same job as the canonical coordinates. For instance, this will be the case, when we later deal with the $C^{\infty}$ convergence of the Bergmann metrics.

Definition 2.1. Let $f(x)$ be a smooth function defined in a neighborhood of zero, if Taylor series of $f(x)$ at $x=0$ converge in a neighborhood of 0 , then $f(x)$ is called weakly real analytic around $0 . f(x)$ is called weakly real analytic function, if it is weakly real analytic at every point.

Clearly, real analytic functions are weakly real analytic., Following example is weakly real analytic at 0 , but not real analytic.

\section{Example.}

$$
f(x)=e^{-\frac{1}{x^{2}}} \text { at } x=0
$$

Of course, it is an interesting question to see what kind of smooth functions are weakly real analytic. In the following, we will construct a smooth function, which is not weakly real analytic.

Example.

$$
f(x)=\sum_{n=0}^{\infty} \frac{1}{\left(i b_{n}\right)^{n-1}} e^{i b_{n} x}
$$


where

$$
b_{n}>\operatorname{Max}\left(2 \sum_{m=0}^{n-1}\left(b_{m}\right)^{n-m+1},(2 n) !\right)
$$

With this assumption for $b_{n}$, we have

$$
\begin{aligned}
f^{(n)}(x)=\sum_{m=0}^{\infty}\left(i b_{m}\right)^{n-m+1} e^{i b_{m} x} \\
\left|f^{(n)}(x)\right| \leq\left|\sum_{m=0}^{n}\left(i b_{m}\right)^{n-m+1} e^{i b_{m} x}\right|+\left|\sum_{m=0}^{\infty} \frac{1}{\left(i b_{n+m+1}\right)^{m}} e^{i b_{n+m+1} x}\right| \\
\leq\left|\sum_{m=0}^{n}\left(i b_{m}\right)^{n-m+1} e^{i b_{m} x}\right|+2
\end{aligned}
$$

Therefore $f(x)$ is smooth. On the other hand

$$
\begin{aligned}
\left|f^{(n)}(x)\right| & \geq b_{n}-\left|\sum_{m=0}^{n-1}\left(i b_{m}\right)^{n-m+1} e^{i b_{m} x}\right|-\left|\sum_{m=0}^{\infty} \frac{1}{\left(i b_{n+m+1}\right)^{m}} e^{i b_{n+m+1} x}\right| \\
& \geq b_{n}-\sum_{m=0}^{n-1}\left(b_{m}\right)^{n-m+1}-\sum_{m=n+1}^{\infty} \frac{1}{((2 m) !)^{m-n-1}} \\
& \geq n^{n} n !
\end{aligned}
$$

Hence

$$
\left|\frac{f^{(n)}(x)}{n !}\right| \geq n^{n}
$$

Since

$$
\sum_{n=0}^{\infty} n^{n} x^{n}
$$

is not convergent any where except at $x=0, f(x)$ is not weakly real analytic at any point, but it is smooth on the whole real line.

Now we assume that $\omega$ is an integer class, then there is an ample Hermitian line bundle $(L, h)$ over $(M, \omega)$ such that

$$
\omega=-\partial \bar{\partial} \log h
$$

There exists a unique section $e_{x}(z)$ of $\left.L\right|_{U_{x}}$ up to circle action such that $\left|e_{x}(0)\right|_{h}=1$ and

$$
h\left(e_{x}(z), e_{x}(z)\right)=\exp \left(-K_{x}\right)
$$


It is very interesting to look at the Kähler-Einstein metrics. When $g$ is Kähler-Einstein ,

$$
R i c_{g}=-\partial \bar{\partial} \log (\operatorname{det} g)=\lambda g
$$

Since $(l, 0),(0, l)$ terms of $g$ vanish, $(l, 1),(1, l)$ terms of $\operatorname{det} g$ vanish, although, presumably only $(l, 0),(0, l)$ terms of $g$ vanish. Therefore, the natural local holomorphic section of canonical bundle of $M$ induced by determinant of the natural flat coordinate is exactly the unique section we discussed above for canonical bundle of $M$. The vanishing of $(l, 1),(1, l)$ terms of $\operatorname{det} g$ merely reflect the fact that any trace with respect to $g$ of any $(l, 2),(2, l)$ terms of $K_{x}$ is zero.

Remark 2.3. One thing we should keep in mind is that no positive definiteness of $\omega$ is required, so we may get affine coordinates with respect to any closed non-degenerate real analytic $(1,1)$ form, which is usually called pseudo-Kähler metric.

Remark 2.4. Clearly all the $(l, k)$ components for $l, k>2$ of $K_{x}$ are independent, they describe the local freedoms of Kähler manifolds. While for $(1,1)$ forms in $\mathbf{C}^{\mathbf{n}}$ the local freedom are characterized by $(l, k)$ components for $l, k \geq 2$.

\section{Tian's peak sections and Bergmann metrics.}

The appearance of K-coordinate reminded me of an old paper of Tian's on Bergmann metric. In that paper, for a projective algebraic Kähler manifold $(M, g)$ with the associated ample Hermitian line bundle $(L, h)$ on $(M, g)$, he considered $L^{m}$ embedding $\varphi_{m}: M \longrightarrow \mathbf{C P}^{\mathbf{N}_{\mathbf{m}}}$, which is induced by an orthonormal basis $\left\{S_{0}^{m}, \cdots, S_{N_{m}}^{m}\right\}$ of the space $H^{0}\left(M, L^{m}\right)$ of all holomorphic global sections of $L^{m}$. Here the inner product on $H^{0}\left(M, L^{m}\right)$ is the natural one induced by the Kähler metric $g$ and the Hermitian metric $h^{m}$ on $L^{m}$, i.e., $\left\langle S_{\alpha}^{m}, S_{\beta}^{m}\right\rangle=\int_{M} h^{m}\left(S_{\alpha}^{m}, S_{\beta}^{m}\right) d V_{g}$. Let $g_{F S}$ be the standard Fubini-Study metric on $\mathbf{C P}^{\mathbf{N}_{\mathrm{m}}}$, i.e., $\omega_{g_{F S}}=\frac{\sqrt{-1}}{2} \partial \bar{\partial} \log \left(\sum_{i=0}^{N_{m}}\left|w_{i}\right|^{2}\right)$. The pullback of the $\frac{1}{m}$-multiple of $g_{F S}$ on $\mathbf{C P}^{\mathbf{N}_{\mathbf{m}}}, g_{m}=\frac{1}{m} \varphi_{m}^{*} g_{F S}$ on $M$, is in the same Kähler class as $g$. It is called the Bergmann metric with respect to $L^{m}$.

It is important to notice that the Bergmann metric $g_{m}$ does not depend on the special choice of the orthonormal basis $\left\{S_{0}^{m}, \cdots, S_{N_{m}}^{m}\right\}$. The main result in that paper is: 
Theorem 3.1 (Tian).

$$
\left\|g_{m}-g\right\|_{C^{2}}=O\left(\frac{1}{\sqrt{m}}\right)
$$

i.e. induced Bergmann metrics are $C^{2}$ convergent to the original metric.

The main point is to construct a sequence of peak sections of $L^{m}$ around a point using Hörmander $L^{2}$-estimate of $\bar{\partial}$-operator. Tian used a coordinate which is a lower order approximation of $\mathrm{K}$-coordinate. The idea is in the local K-coordinate the canonical section $e_{x}(z)$ of $L$ has norm

$$
\begin{gathered}
\left\|e_{x}(z)\right\|=\exp \left(-|z|^{2}+\frac{1}{4} R_{i \bar{j} k \bar{l}} z^{i} z^{\bar{j}} z^{k} z^{\bar{l}}+O\left(|z|^{5}\right)\right) \\
\left\|e_{x}^{m}(z)\right\|=\exp \left(-m\left(|z|^{2}+O\left(|z|^{4}\right)\right)\right)
\end{gathered}
$$

which is roughly the rapidly degenerating Gaussian function. When $m$ is large, it is very easy to smooth out and use Hörmander $L^{2}$-estimate of $\bar{\partial}$-operator to create a global holomorphic section that roughly looks like $e_{x}^{m}(z)$, which have the property that it is like a peak at $m$ and almost zero away from $m$.

One can actually create a sequence of sections with similar nature, such that for $P=\left(p_{1}, p_{2}, \cdots p_{n}\right)$

$$
e_{P} \sim z_{1}^{p_{1}} z_{2}^{p_{2}} \cdots z_{n}^{p_{n}} e_{x}^{m}
$$

Tian then went on using this sections to show his theorem. Due to the complexities that arise when order get higher, he only showed convergence for $C^{2}$, although he believed higher order estimate should be possible with a more neat method.

We will analyze the situation more closely and explore the $C^{\infty}$ convergence. First, let's fix some notations and summarize some known results.

For the notations, we will use the following convention, here letter "P" is just for illustration. We will also use other letters with similar convention.

For $P=\left(p_{1}, p_{2}, \cdots, p_{n}\right) \in \mathbf{Z}_{+}^{\mathbf{n}}$, let

$$
p=\sum_{i=1}^{n} p_{i}, \quad P !=\prod_{i=1}^{n} p_{i} !
$$


For $z=\left(z^{1}, z^{2}, \cdots, z^{n}\right) \in \mathbf{C}^{\mathbf{n}}$, let

$$
\begin{gathered}
d z=d z^{1} \wedge d z^{2} \wedge \cdots \wedge d z^{n}, \quad d \bar{z}=d \bar{z}^{1} \wedge d \bar{z}^{2} \wedge \cdots \wedge d \bar{z}^{n} \\
d z \wedge d \bar{z}=\bigwedge_{i=1}^{n} d z^{i} \wedge d \bar{z}^{i}, \quad z^{P}=\left(z^{1}\right)^{p_{1}}\left(z^{2}\right)^{p_{2}} \cdots\left(z^{n}\right)^{p_{n}} \\
\partial_{P}=\left(\frac{\partial}{\partial z^{1}}\right)^{p_{1}}\left(\frac{\partial}{\partial z^{2}}\right)^{p_{2}} \cdots\left(\frac{\partial}{\partial z^{n}}\right)^{p_{n}}
\end{gathered}
$$

The construction of peak section in Tian's paper is well done, we really have no more to say on this. We simply state it as follows.

Lemma 3.1 (Tian). For an $n$-tuple of integers $P=\left(p_{1}, p_{2}, \cdots, p_{n}\right) \in \mathbf{Z}_{+}^{\mathbf{n}}$ and an integer $p^{\prime}>p=p_{1}+p_{2}+\cdots+p_{n}$, there exists an $m_{0}>0$ such that for $m>m_{0}$, there is a holomorphic global section $S_{P}$ in $H^{0}\left(M, L^{m}\right)$, satisfying

$$
\begin{gathered}
\int_{M}\left\|S_{P}\right\|_{h^{m}}^{2} d V_{g}=1, \\
\int_{M \backslash\left\{\rho(z) \leq \frac{\log m}{\sqrt{m}}\right\}}\left\|S_{P}\right\|_{h^{m}}^{2} d V_{g}=O\left(\frac{1}{m^{2 p^{\prime}}}\right),
\end{gathered}
$$

and locally at $x_{0}$,

$$
S_{P}(z)=\lambda_{P}\left(z^{P}+O\left(|z|^{2 p^{\prime}}\right)\right) e_{x}^{m}\left(1+O\left(\frac{1}{m^{2 p^{\prime}}}\right)\right)
$$

where $\|\cdot\|_{h^{m}}$ is the norm on $L^{m}$ given by $h^{m}$, and $O\left(1 / m^{2 p^{\prime}}\right)$ denotes a quantity dominated by $C / m^{2 p^{\prime}}$ with the constant $C$ depending only on $p^{\prime}$ and the geometry of $M$, moreover

$$
\lambda_{P}^{-2}=\int_{\rho(z) \leq \frac{\log m}{\sqrt{m}}}\left|z^{P}\right|^{2} a^{m} d V_{g}
$$

where $d V_{g}=\operatorname{det}\left(g_{i \bar{j}}\right)(\sqrt{-1} /(2 \pi))^{n} d z \wedge d \bar{z}$ is the volume form.

For the estimates of the inner products between peak sections, we need the following straightforward generalization of Tian's.

Lemma 3.2. Let $S_{P}(z)=\left(1+O\left(\frac{1}{m^{2 p^{\prime}}}\right)\right)\left(z^{P}+O\left(|z|^{2 p^{\prime}}\right)\right) e_{L}^{m}$ be the section constructed as above. $T$ be another section of $L^{m}$. 
(i) If $z^{P}$ is not in T's Taylor expansion, then

$$
\left\langle S_{P}, T\right\rangle_{h^{m}}=O\left(\frac{1}{m}\right)\left\|S_{P}\right\|_{h^{m}}\|T\|_{h^{m}} .
$$

(ii) If $T$ contains no terms $z^{Q}$, such that $q<p+d(d \geq 1)$ then

$$
\left\langle S_{P}, T\right\rangle_{h^{m}}=O\left(\frac{1}{m^{1+d / 2}}\right)\left\|S_{P}\right\|_{h^{m}}\|T\|_{h^{m}} .
$$

Proof. We will only show (ii), $(i)$ is simpler.

Assume that

$$
e^{m\left(|z|^{2}-K(z, \bar{z})\right)} \operatorname{det} g=\sum_{S, T} a_{S \bar{T}} z^{S} \bar{z}^{T}
$$

Then

$$
\begin{aligned}
\left\langle S_{P}, T\right\rangle_{h^{m}}= & \int_{|z| \leq \frac{\log m}{\sqrt{m}}} z^{P \overline{f_{T}}}(z) e^{-m|z|^{2}}\left(\sum_{S, Q} a_{S \bar{Q}} z^{S} \bar{z}^{Q}\right) d z d \bar{z}+O\left(\frac{1}{m^{p^{\prime}}}\right) \\
= & \int_{|z| \leq \frac{\log m}{\sqrt{m}}} z^{P \overline{f_{T}}}(z) e^{-m|z|^{2}}\left(\sum_{s-q<d} a_{S \bar{Q}} z^{S} \bar{z}^{Q}\right) d z d \bar{z} \\
& +\int_{|z| \leq \frac{\operatorname{logm}}{\sqrt{m}}} z^{P \overline{f_{T}}}(z)\left(O\left(m|z|^{d+4}+|z|^{d+2}\right)\right) h^{m}(z) d V_{g} \\
\leq & C\left[\int_{|z| \leq \frac{\operatorname{logm}}{\sqrt{m}}}\left|z^{P}\right|^{2}\left(m^{2}|z|^{2 d+8}+|z|^{2 d+4}\right) h^{m}(z) d V_{g}\right]^{\frac{1}{2}}\|T\|_{h^{m}} \\
= & O\left(\frac{1}{m^{\frac{d+2}{2}}}\right)\left\|S_{P}\right\|_{h^{m}}\|T\|_{h^{m}}
\end{aligned}
$$

Now we will compute inner products of $S_{P}$. Here we make a modification, replacing Tian's factor $\left(1-|z|^{2}\right)^{m}$ by $e^{-m|z|^{2}}$ which seems more natural and easier to compute.

\section{Lemma 3.3.}

$\left(\frac{\sqrt{-1}}{2}\right)^{n} \int_{\rho(z) \leq \frac{\log m}{\sqrt{m}}}\left|z^{P}\right|^{2} e^{-m|z|^{2}} d z \wedge d \bar{z}=\left(\frac{\pi}{m}\right)^{n} \frac{P !}{m^{p}}+O\left(\frac{1}{m^{2 p^{\prime}}}\right)$ for $m$ large 
Proof. It is easy to see that it will not make a difference, if we extend domain of integration to $\mathbf{C}^{\mathbf{n}}$. Then the integral is of separated variables and result is a corollary of the fact:

$$
\int_{0}^{\infty} r^{2 p} e^{-m r^{2}} r d r=\frac{1}{2} \frac{p !}{m^{p}}
$$

Now recall that

$$
e^{m\left(|z|^{2}-K(z, \bar{z})\right)} \operatorname{det} g=\sum_{S, T} a_{S \bar{T}} z^{S} \bar{z}^{T} .
$$

Then we have the following lemma.

\section{Lemma 3.4.}

$$
\left\langle S_{P}, S_{Q}\right\rangle_{h^{m}}=\left(\frac{\pi}{m}\right)^{n} \sum_{S+P=Q+T} a_{S \bar{T}} \frac{(S+P) !}{m^{s+p}} .
$$

Especially

$$
\left\|S_{P}\right\|_{h^{m}}^{2}=\left(\frac{\pi}{m}\right)^{n} \sum_{S} a_{S \bar{S}} \frac{(S+P) !}{m^{s+p}}
$$

with the leading term

$$
\left(\frac{\pi}{m}\right)^{n} \frac{P !}{m^{p}}
$$

Notice:

$$
\begin{gathered}
\omega_{m}=\frac{\sqrt{-1}}{2 \pi} \partial \bar{\partial} \frac{1}{m} \log \left(\sum_{i=0}^{N_{m}}\left|S_{i}^{m}\right|^{2}\right) \\
\omega_{g}=\frac{\sqrt{-1}}{2 \pi} \partial \bar{\partial} K
\end{gathered}
$$

here

$$
K=|z|^{2}-\frac{1}{4} R_{i \bar{j} k \bar{l}} z^{i} \bar{z}^{j} z^{k} \bar{z}^{l}+O\left(|z|^{5}\right)
$$

For us it is important to notice that $\omega_{m}$ converge to $\omega_{g}$ in $C^{\infty}$ is equivalent to that the Kähler potentials $K_{m}=\frac{1}{m} \log \left(\sum_{i=0}^{N_{m}}\left|S_{i}^{m}\right|^{2}\right)$ converge to $K$ in $C^{\infty}$. This is what we will try to show.

First put index $P$ in an order. Let $|P|$ denote its order, then we can compare different indices according to this order. The idea is if we care 
about the $z^{P}$ term, we want to take a $D \gg P$ and also $m \gg d, p^{\prime} \gg d$ such that, we can just use $S_{P}, P \leq D$ to do the computation and be able to ignore terms $>D$. This need a very careful arrangement.

First construct a filtration of $H^{0}\left(M, L^{m}\right)$, let

$$
V_{P}=\left\{S \in H^{0}\left(M, L^{m}\right):\left.\partial_{Q} S\right|_{m}=0 \text { for } Q<P\right\}
$$

If $Q>P$ is "smallest", then $\operatorname{dim}\left(V_{Q} / V_{P}\right) \leq 1$.

We can then associate an orthonormal basis of $H^{0}\left(M, L^{m}\right)$ with respect to this filtration, denote by $\left\{S_{0}^{m}, \cdots, S_{N_{m}}^{m}\right\}$. We replace this by $\left\{T_{0}, T_{e_{1}}, \cdots T_{D}, S_{|D|+1}^{m}, \cdots, S_{N_{m}}^{m}\right\}$ where $T_{P}=S_{P} /\left\|S_{P}\right\|_{h^{m}}$.

We intend to make them orthogonal again, but in a special way. We first modify $T_{P}$ for $P \leq D$ to make them orthogonal to $S_{i}^{m}$ for $i>|D|$. By which we get

$$
\tilde{T}_{P}=T_{P}+\sum_{j=|D|+1}^{N_{m}} \beta_{P j} S_{j}^{m}
$$

Use lemma 3.2 for $S_{P}$ and $\sum_{j=|D|+1}^{N_{m}} \beta_{P j} S_{j}^{m}$, we get

$$
\sum_{j=|D|+1}^{N_{m}}\left|\beta_{P j}\right|^{2}=O\left(m^{-(d+3-p)}\right)
$$

Now we try to rectify $\left\{\tilde{T}_{P}\right\}$. Our aim is to rule out the influence of $\left\{S_{j}^{m}\right\}_{j=|D|+1}^{N_{m}}$. We will find out, after all, if we care $z^{P}$ term, the influence of $\left\{S_{j}^{m}\right\}$ only reflect on the coefficients of $\beta_{Q R}, Q, R<P$. Since all the other terms vanish at $x_{0}$. Now we try to trace the influence of $\left\{S_{j}^{m}\right\}$ on $\beta_{Q R}$. Rectifying $\left\{\tilde{T}_{P}\right\}$ requires operations similar to

$$
\tilde{T}_{P}-\frac{\left\langle\tilde{T}_{P}, \tilde{T}_{Q}\right\rangle}{\left|\tilde{T}_{P}\right|\left|\tilde{T}_{Q}\right|} \tilde{T}_{Q}
$$

where $Q>P$. The major terms coming from $\left\{S_{j}^{m}\right\}$ is

$$
\begin{gathered}
-\left\langle T_{P}, \sum_{j=|D|+1}^{N_{m}} \beta_{Q j} S_{j}^{m}\right\rangle-\left\langle T_{Q}, \sum_{j=|D|+1}^{N_{m}} \beta_{P j} S_{j}^{m}\right\rangle \\
+\frac{1}{2}\left\langle T_{P}, T_{Q}\right\rangle\left\langle T_{Q}, \sum_{j=|D|+1}^{N_{m}} \beta_{Q j} S_{j}^{m}\right\rangle
\end{gathered}
$$


which is of order $O\left(m^{\frac{p+q}{2}-(3+d)}\right)$. A careful analysis will show that other terms are of lower order and can be ignored.

Assume that after rectification, we get

$$
\hat{T_{P}}=T_{P}+\sum_{P<Q \leq D} \beta_{P Q} T_{Q}+\sum_{j=|D|+1}^{N_{m}} \beta_{P j} S_{j}^{m}
$$

For simplicity, here we still use the same notation for $\beta_{P j}$, although it have been changed. It is easy to see its order is unchanged. Then we have

$$
\beta_{P Q}=\gamma_{P Q}+O\left(m^{\frac{p+q}{2}-(3+d)}\right)
$$

Here $\gamma_{P Q}$ comes from pure interaction of $\left\{T_{P}\right\}$.

We are interested in

$$
\frac{1}{m} \log \left(\sum_{i=0}^{N_{m}}\left|S_{i}^{m}\right|^{2}\right)=\frac{1}{m} \log \left(\sum_{0 \leq Q \leq D} \frac{\left|\hat{T}_{Q}\right|^{2}}{\left\|\hat{T}_{Q}\right\|_{h^{m}}^{2}}+\sum_{i=|D|+1}^{N_{m}}\left|S_{i}^{m}\right|^{2}\right)
$$

When we worry about $z^{P}$ term, only $\left|\hat{T}_{Q}\right|^{2} /\left\|\hat{T}_{Q}\right\|_{h^{m}}^{2}$ terms for $Q \leq P$ are involved. A careful analysis will reveal that $\left\|\hat{T}_{Q}\right\|_{h^{m}}^{2}$ terms have error terms controlled by $O\left(m^{p-(4+d)}\right)$, and the $\left|\hat{T}_{Q}\right|^{2}$ terms have error terms controlled by $O\left(m^{p-(3.5+d)}\right)$. They are all negligible, since $d \gg p$.

Above argument ensure that we only need to work with the subspace of $H_{0}\left(M, L^{m}\right)$ generated by $\left\{S_{P}\right\}_{p \leq d}$. Another very crucial observation is that although orthonormal basis is good for abstract arguments, it is not suitable for computation. We will use the more natural but non-orthogonal basis $\left\{S_{P}\right\}$ to do the computation.

Let $\left\{T_{i}^{m}\right\}$ be a not necessarily orthonormal basis of $H^{0}\left(M, L^{m}\right)$, and $\left\{S_{i}^{m}\right\}$ be the original orthonormal basis. Then

$$
\left|S_{0}^{m}\right|^{2}+\left|S_{1}^{m}\right|^{2}+\cdots\left|S_{N_{m}}^{m}\right|^{2}=\sum_{i, j=0}^{N_{m}} G^{i \bar{j}} T_{i}^{m} \overline{T_{j}^{m}}
$$

Here $G^{i \bar{j}} G_{k \bar{j}}=\delta_{i k}, G_{i \bar{j}}=\left\langle T_{i}^{m}, T_{j}^{m}\right\rangle_{h^{m}}$.

So we only need to compute

$$
\frac{1}{m} \log \left(\sum_{P, Q} G^{P \bar{Q}} S_{P} \overline{S_{Q}}\right)
$$


Here $G^{P \bar{Q}} G_{R \bar{Q}}=\delta_{P R}, G_{P \bar{Q}}=\left\langle S_{P}, S_{Q}\right\rangle_{h^{m}}$.

Let Kähler potential be

$$
K(z, \bar{z})=|z|^{2}+\sum_{P, Q} c_{P \bar{Q}^{P}} z^{P} \bar{z}^{Q}
$$

What we expect is that all $m^{r}$ terms for $\frac{1}{m} \log \left(\sum_{P, Q} G^{P \bar{Q}} S_{P} \overline{S_{Q}}\right)$ of $r>0$ vanish and the terms that do not involve $m$ should equal to $K(z, \bar{z})$.

Notice that $\left\{c_{P \bar{Q}}\right\}$ are independent, so we may trace them independently. Now we have reduced our problem into a purely combinatoric one. In principle, the problem is already solved, if one is optimistic. But it turns out the corresponding combinatoric problem is extremely complicated. Although we are able to sort out almost all combinatorics, the complete solution of the problem needs to go back to some geometrical arguments.

\section{Combinatorics Involved.}

In this section we will demonstrate the combinatorics involved, and try to solve it to a great extent in next section.

As we mentioned before that $\left\{c_{P \bar{Q}}\right\}$ are independent, so without loss of generality, we only need to consider finitely many terms. Let Kähler potential be

$$
K(z, \bar{z})=|z|^{2}+\sum_{i=1}^{r} c_{P^{i} \bar{Q}^{i}} z^{P^{i}} \bar{z}^{Q^{i}}
$$

then

$$
g_{i \bar{j}}=\delta_{i j}+\sum_{k=1}^{r} p_{, i}^{k} q_{, j}^{k} c_{P^{k}} \bar{Q}^{k} z^{P^{k}-e_{i}} \bar{z}^{Q^{k}-e_{j}}
$$


and

$$
\begin{aligned}
& \operatorname{det}(g)=1+\sum_{i=1}^{r} \sum_{j=1}^{n} p_{, j}^{i} q_{, j}^{i} c_{P^{i} \bar{Q}^{i}} z^{P^{i}-e_{j}} \bar{z}^{Q^{i}-e_{j}}+\cdots \\
& =1+\sum_{i=1}^{r} c_{P^{i} \bar{Q}^{i}} z^{P^{i}} \bar{z}^{Q^{i}} \sum_{j=1}^{n}\left(\frac{p_{, j}^{i}}{z^{j}}\right)\left(\frac{q_{, j}^{i}}{\bar{z}^{j}}\right)
\end{aligned}
$$

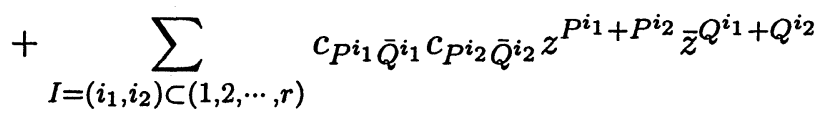

$$
\begin{aligned}
& \sum_{\left.j_{2}\right) \subset(1,2, \cdots, n)} \frac{\operatorname{det}\left(P_{, J}^{I}\right)}{z^{J}} \frac{\operatorname{det}\left(Q_{, J}^{I}\right)}{\bar{z}^{J}}+\cdots \\
& =\sum_{I \subset(1,2, \cdots, r)}\left(\prod_{i \in I} c_{P^{i} \bar{Q}^{i}} z^{P^{i}} \bar{z}^{Q^{i}}\right) \sum_{J \subset(1,2, \cdots, n)} \frac{\operatorname{det}\left(P_{, J}^{I}\right)}{z^{J}} \frac{\operatorname{det}\left(Q_{, J}^{I}\right)}{\bar{z}^{J}}
\end{aligned}
$$

Recall

$$
e^{m\left(|z|^{2}-K(z, \bar{z})\right)} \operatorname{det} g=\sum_{S, T} \tilde{a}_{S \bar{T}} z^{S} \bar{z}^{T} .
$$

Here we use $\tilde{a}_{S \bar{T}}$ instead of $a_{S \bar{T}}$ which was used. Let

$$
b_{P \bar{Q}}=-c_{P \bar{Q}} m^{1-\frac{p+q}{2}}, \quad a_{P \bar{Q}}=\tilde{a}_{P \bar{Q}} m^{-\frac{p+q}{2}}
$$

Then

$$
\begin{aligned}
& \sum_{S, T} a_{S \bar{T}}(\sqrt{m} z)^{S}(\sqrt{m} \bar{z})^{T}=\sum_{S, T} \tilde{a}_{S \bar{T}} z^{S} \bar{z}^{T}=e^{m\left(|z|^{2}-K(z, \bar{z})\right)} \operatorname{det} g \\
& =e^{-m\left(\sum_{i=1}^{r} c_{P^{i} \bar{Q}^{i}} P^{P^{i}} \bar{z}^{Q^{i}}\right)}\left(1+\sum_{i=1}^{r} c_{P^{i} \bar{Q}^{i}} \sum_{j=1}^{n} p_{, j}^{i} q_{, j}^{i} z^{P^{i}-e_{j}} \bar{z}^{Q^{i}-e_{j}}+\cdots\right) \\
& =\sum_{P, Q}\left(\begin{array}{l}
\sum_{\substack{L=\left(l_{1}, l_{2}, \cdots l_{r}\right) \\
\sum_{i=1}^{r} l_{i}\left(P^{i}, Q^{i}\right)=(P, Q)}} \frac{1}{L !} \prod_{i=1}^{r} b_{P^{i} \bar{Q}^{i}}^{l_{i}}
\end{array}\right)(\sqrt{m} z)^{P}(\sqrt{m} \bar{z})^{Q} \\
& \left(1-\sum_{i=1}^{r} b_{P^{i} \bar{Q}^{i}} \sum_{j=1}^{n} p_{, j}^{i} q_{, j}^{i}(\sqrt{m} z)^{P^{i}-e_{j}}(\sqrt{m} \bar{z})^{Q^{i}-e_{j}}+\cdots\right)
\end{aligned}
$$




$$
\begin{aligned}
& =\sum_{P, Q}\left(\begin{array}{c}
\sum_{\substack{L=\left(l_{1}, l_{2}, \cdots l_{r}\right) \\
\sum_{i=1}^{r} l_{i}\left(P^{i}, Q^{i}\right)=(P, Q)}} \frac{1}{L !} \prod_{i=1}^{r} b_{P^{i} \bar{Q}^{i}}^{l_{i}} \\
\left.\sum^{i}\right)
\end{array}\right)(\sqrt{m} z)^{P}(\sqrt{m} \bar{z})^{Q} \\
& \sum_{I \subset(1,2, \cdots, r)}(-1)^{|I|}\left(\prod_{i \in I} b_{P^{i} \tilde{Q}^{i}}(\sqrt{m} z)^{P^{i}}(\sqrt{m} \bar{z})^{Q^{i}}\right) \\
& \sum_{J \subset(1,2, \cdots, n)} \frac{\operatorname{det}\left(P_{, J}^{I}\right)}{(\sqrt{m} z)^{J}} \frac{\operatorname{det}\left(Q_{, J}^{I}\right)}{(\sqrt{m} \bar{z})^{J}}
\end{aligned}
$$

Let $S_{P}=z^{P} e_{L}^{m}$ be the sections we constructed. Then according to lemma 3.4 we have

$$
\left\langle S_{P}, S_{Q}\right\rangle_{h^{m}}=\left(\frac{\pi}{m}\right)^{n} \sum_{S+P=Q+T} \tilde{a}_{S \bar{T}} \frac{(S+P) !}{m^{s+p}} .
$$

We define

$$
G_{S \bar{T}}=\sum_{S+P=Q+T} \tilde{a}_{P \bar{Q}} \frac{(S+P) !}{m^{s+p}}=\frac{S !}{m^{\frac{s+t}{2}}}\left(\sum_{S+P=Q+T} a_{P \bar{Q}} \frac{(S+P) !}{S !}\right)
$$

Let

$$
\Lambda_{S \bar{T}}=\frac{S !}{m^{s}} \delta_{S T} \Lambda^{S \bar{T}}=\frac{m^{s}}{S !} \delta_{S T}
$$

Then

$$
G_{S \bar{T}}=\Lambda_{S \bar{T}}+G_{S \bar{T}}^{\prime}
$$

To compute inverse of matrix $G=\left(G_{S \bar{T}}\right)=\Lambda+G^{\prime}$, we use the following formula.

$$
G^{-1}=\Lambda^{-1}-\Lambda^{-1} G^{\prime} \Lambda^{-1}+\left(\Lambda^{-1} G^{\prime}\right)^{2} \Lambda^{-1}+\cdots
$$

Use this equality we get

$$
\begin{aligned}
G^{T \bar{S}}= & \Lambda^{S \bar{T}}+\frac{m^{\frac{s+t}{2}}}{S !} \sum_{u=1}^{\infty}(-1)^{u} \sum_{\sum_{j=1}^{u} Q_{j}-P_{j}=S-T} \\
& \left(\prod_{j=1}^{u} a_{P_{j} \bar{Q}_{j}}\right)\left(\prod_{j=1}^{u} \frac{\left(S+\sum_{i=1}^{j}\left(P_{i}-Q_{i}\right)+Q_{j}\right) !}{\left(S+\sum_{i=1}^{j}\left(P_{i}-Q_{i}\right)\right) !}\right)
\end{aligned}
$$


Here $Q_{j}, P_{j} \in \mathbf{Z}_{+}^{\mathbf{n}}$.

To write in terms of $b$, we recall,

$$
\begin{aligned}
& \sum_{S, T} a_{S \bar{T}}(\sqrt{m} z)^{S}(\sqrt{m} \bar{z})^{T}=\sum_{S, T} \tilde{a}_{S \bar{T}} z^{S} \bar{z}^{T} \\
& =\sum_{P, Q}\left(\sum_{\substack{L=\left(l_{1}, l_{2}, \cdots l_{r}\right) \\
\sum_{i=1}^{r} l_{i}\left(P^{i}, Q^{i}\right)=(P, Q)}} \frac{1}{L !} \prod_{i=1}^{r} b_{P^{i} \bar{Q}^{i}}^{l_{i}}\right)(\sqrt{m} z)^{P}(\sqrt{m} \bar{z})^{Q} \\
& \left(1-\sum_{i=1}^{r} b_{P^{i} \bar{Q}^{i}} \sum_{j=1}^{n} p_{, j}^{i} q_{, j}^{i}(\sqrt{m} z)^{P^{i}-e_{j}}(\sqrt{m} \bar{z})^{Q^{i}-e_{j}}+\cdots\right) \\
& =\sum_{P, Q}\left(\sum_{\substack{L=\left(l_{1}, l_{2}, \cdots l_{r}\right) \\
\sum_{i=1}^{r} l_{i}\left(P^{i}, Q^{i}\right)=(P, Q)}} \frac{1}{L !} \prod_{i=1}^{r} b_{P^{i} \bar{Q}^{i}}^{l_{i}}\right)(\sqrt{m} z)^{P}(\sqrt{m} \bar{z})^{Q} \\
& \sum_{i=1}^{r} \sum_{j=1}^{n} p_{, j}^{i} q_{, j}^{i} \frac{1}{\left(L-e_{i}\right) !}(\sqrt{m} z)^{P-e_{j}}(\sqrt{m} \bar{z})^{Q-e_{j}} \ldots \\
& =\sum_{P, Q} \sum_{\substack{L=\left(l_{1}, l_{2}, \cdots l_{r}\right) \\
\sum_{i=1}^{r}}}\left(\prod_{\prod^{i}}^{r} b_{P^{i} \bar{Q}^{i}}^{l_{i}}\right) \\
& \sum_{i=1}^{r} l_{i}\left(P^{i}, Q^{i}\right)=(P, Q) \\
& \sum_{I \subset(1,2, \cdots, r)} \sum_{J \subset(1,2, \cdots, n)} \operatorname{det}\left(P_{, J}^{I}\right) \operatorname{det}\left(Q_{, J}^{I}\right) \\
& \frac{(-1)^{|I|}}{\left(L-e_{I}\right) !}(\sqrt{m} z)^{P-e_{J}}(\sqrt{m} \bar{z})^{Q-e_{J}}
\end{aligned}
$$


which gives

$$
\begin{aligned}
& a_{P \bar{Q}}=\left(\begin{array}{c}
\sum_{\substack{L=\left(l_{1}, l_{2}, \cdots l_{r}\right) \\
\sum_{i=1}^{r} l_{i}\left(P^{i}, Q^{i}\right)=(P, Q)}} \frac{1}{L !} \prod_{i=1}^{r} b_{P^{i} \bar{Q}^{i}}^{l_{i}}
\end{array}\right) \\
& -\sum_{j=1}^{n} \sum_{L=\left(l_{1}, l_{2}, \cdots l_{r}\right)} \quad\left(\prod_{i=1}^{r} b_{P^{i} \bar{Q}^{i}}^{l_{i}}\right) \\
& \sum_{i=1}^{r} l_{i}\left(P^{i}, Q^{i}\right)=\left(P+e_{j}, Q+e_{j}\right) \\
& \sum_{i=1}^{r} p_{, j}^{i} q_{, j}^{i} \frac{1}{\left(L-e_{i}\right) !}+\cdots \\
& =\sum_{J \subset(1,2, \cdots, n)} \\
& \sum \\
& L=\left(l_{1}, l_{2}, \cdots l_{r}\right) \\
& \sum_{i=1}^{r} l_{i}\left(P^{i}, Q^{i}\right)=\left(P+e_{J}, Q+e_{J}\right) \\
& \sum_{I \subset(1,2, \cdots, r)} \operatorname{det}\left(P_{, J}^{I}\right) \operatorname{det}\left(Q_{, J}^{I}\right) \frac{(-1)^{|I|}}{\left(L-e_{I}\right) !}
\end{aligned}
$$

Then

$$
\begin{aligned}
& G^{T \bar{S}}=\Lambda^{S \bar{T}}+\frac{m^{\frac{s+t}{2}}}{S !} \sum_{u=1}^{\infty}(-1)^{u} \quad \sum_{L=\left(l_{1}, l_{2}, \cdots l_{r}\right)} \quad \frac{1}{\prod_{j=1}^{u}\left(L^{j}\right) !} \\
& \sum_{j=1}^{u} L^{j}=L \\
& \sum_{i=1}^{r} l_{i}\left(P^{i}-Q^{i}\right)=T-S \\
& \left(\prod_{i=1}^{r} b_{P^{i} \bar{Q}^{i}}^{l_{i}}\right)\left(\prod_{j=1}^{u} \frac{\left(S+\sum_{i=1}^{j}\left(P_{i}-Q_{i}\right)+Q_{j}\right) !}{\left(S+\sum_{i=1}^{j}\left(P_{i}-Q_{i}\right)\right) !}\right) \\
& \left(1-\sum_{j=1}^{u} \sum_{i=1}^{r} \sum_{k=1}^{n} p_{, k}^{i} q_{, k}^{i} l_{i}^{j} \frac{1}{s_{, k}+\sum_{i=1}^{j}\left(p_{i, k}-q_{i, k}\right)+q_{j, k}}+\cdots\right) \\
& =\Lambda^{S \bar{T}}+\frac{m^{\frac{s+t}{2}}}{S !} \sum_{u=1}^{\infty}(-1)^{u} \\
& \sum_{L=\left(l_{1}, l_{2}, \cdots l_{r}\right)} \frac{1}{\prod_{j=1}^{u}\left(L^{j}\right) !} \\
& \sum_{j=1}^{u} L^{j}=L \\
& \sum_{i=1}^{r} l_{i}\left(P^{i}-Q^{i}\right)=T-S
\end{aligned}
$$




$$
\begin{aligned}
& \left(\prod_{i=1}^{r} b_{P^{i} \bar{Q}^{i}}^{l_{i}}\right)\left(\prod_{j=1}^{u} \frac{\left(S+\sum_{i=1}^{j}\left(P_{i}-Q_{i}\right)+Q_{j}\right) !}{\left(S+\sum_{i=1}^{j}\left(P_{i}-Q_{i}\right)\right) !}\right) \\
& \left(\prod_{j=1}^{u} \sum_{\substack{I_{j} \subset(1,2, \cdots, r) \\
K_{j} \subset(1,2, \cdots, n)}} \operatorname{det}\left(P_{, K_{j}}^{I_{j}}\right) \operatorname{det}\left(Q_{, K_{j}}^{I_{j}}\right)\left(L^{j}\right)^{e_{I_{j}}}\right. \\
& \left.\frac{(-1)^{\left|K_{j}\right|}}{\prod_{k \in K_{j}}\left(s_{, k}+\sum_{i=1}^{j}\left(p_{i, k}-q_{i, k}\right)+q_{j, k}\right)}\right)
\end{aligned}
$$

Here $e_{I}=\sum_{i \in I} e_{i}, L^{j}=\left(l_{1}^{j}, l_{1}^{j}, \cdots l_{r}^{j}\right)$, and $P_{j}=\sum_{i=1}^{r} l_{i}^{j} P^{i}, Q_{j}=\sum_{i=1}^{r} l_{i}^{j} Q^{i}$. Let

$$
I_{L}=\sum_{u=1}^{\infty}(-1)^{u} \sum_{\sum_{j=1}^{u} L^{j}=L} \frac{1}{\prod_{j=1}^{u}\left(L^{j}\right) !}
$$

Here $L=\left(l_{1}, l_{1}, \cdots l_{r}\right)$. Notice that

$$
\sum_{L} I_{L} T^{L}=\sum_{u=1}^{\infty}(-1)^{u}\left(e^{t}-1\right)^{u}=e^{-t}=\sum_{L} \frac{(-1)^{l}}{L !} T^{L}
$$

here $T=\left(t_{1}, t_{1}, \cdots t_{r}\right), t=\left(t_{1}+t_{1}+\cdots+t_{r}\right)$. Therefore

$$
I_{L}=\sum_{u=1}^{\infty}(-1)^{u} \sum_{\sum_{j=1}^{u} L^{j}=L} \frac{1}{\prod_{j=1}^{u}\left(L^{j}\right) !}=\frac{(-1)^{l}}{L !}
$$

With this equality in mind, we can write $G^{T \bar{S}}$ as

$$
\begin{gathered}
G^{T \bar{S}}=\Lambda^{S \bar{T}}+\frac{m^{\frac{s+t}{2}}}{S !} \sum_{\substack{L=\left(l_{1}, l_{2}, \cdots l_{r}\right) \\
\sum_{i=1}^{r} l_{i}\left(P^{i}-Q^{i}\right)=T-S}} \frac{\prod_{i=1}^{r}\left(-b_{P^{i} \bar{Q}^{i}}\right)^{l_{i}}}{L !} \\
\left(\frac{S !}{\left(S-\sum_{i=1}^{r} l_{i} Q^{i}\right) !}+\sum_{j=1}^{n} D_{1, j}(L, P, Q) \frac{S !}{\left(S-\sum_{i=1}^{r} l_{i} Q^{i}+e_{j}\right) !}+\cdots\right)
\end{gathered}
$$

Use this expression to compute the Kähler potential, we get

$$
K_{m}=\frac{1}{m} \log \left(1+\sum_{S, T} \frac{G^{T \bar{S}}}{G^{0 \overline{0}}} \bar{z}^{S} z^{T}\right)
$$




$$
\begin{aligned}
=\frac{1}{m} \sum_{k=1}^{\infty} \frac{(-1)^{k-1}}{k} \sum_{S, T} \bar{z}^{S} z^{T} \sum_{\sum_{i=1}^{k} S_{i}=S} \prod_{i=1}^{k} \frac{G^{T_{i} \bar{S}_{i}}}{G^{0 \overline{0}}} \\
\sum_{i=1}^{k} T_{i}=T \\
=\frac{1}{m} \sum_{k=1}^{\infty} \frac{(-1)^{k-1}}{k} \sum_{S, T}(\sqrt{m} z)^{S}(\sqrt{m} \bar{z})^{T} \sum_{\substack{\sum_{i=1}^{k} S_{i}=S \\
\sum_{i=1}^{k} T_{i}=T}}^{\frac{1}{\prod_{i=1}^{k} S_{i} !}}
\end{aligned}
$$

$$
\begin{aligned}
& \sum_{\substack{\left.L=\left(l_{1}, l_{2}, \cdots l_{r}\right) \\
\sum^{r}{ }^{\prime} l_{\left(P^{i}\right.} Q^{i}\right)=T-S}}^{r}\left(-b_{P^{i} \bar{Q}^{i}}\right)^{l_{i}} \sum_{\sum_{i=1}^{k} L^{i}=L} \frac{1}{\prod_{i=1}^{k}\left(L^{i}\right) !} \\
& \sum_{i=1}^{r} l_{i}\left(P^{i}-Q^{i}\right)=T-S \\
& \prod_{i=1}^{k}\left(\frac{S_{i} !}{\left(S_{i}-\sum_{j=1}^{r} l_{j}^{i} Q^{j}\right) !}\right. \\
& \left.\left.+\sum_{v=1}^{n} D_{1, v}\left(L, P_{v}, Q_{v}\right) \frac{S_{i} !}{\left(S_{i}-\sum_{j=1}^{r} l_{j}^{i} Q^{j}+e_{v}\right) !}+\cdots\right)\right] \\
& =\frac{1}{m} \sum_{S-T} \sum_{L=\left(l_{1}, l_{2}, \cdots l_{r}\right)} \prod_{i=1}^{r}\left(-b_{P^{i} \bar{Q}^{i}}\right)^{l_{i}} \\
& \sum_{i=1}^{r} l_{i}\left(P^{i}-Q^{i}\right)=T-S \\
& \sum_{u=1}^{\infty} \sum_{\sum_{i=1}^{u} L^{i}=L} \frac{1}{\prod_{i=1}^{u}\left(L^{i}\right) !} \\
& \sum_{S}(\sqrt{m} z)^{S}(\sqrt{m} \bar{z})^{T} \sum_{k=u}^{\infty} \frac{(-1)^{k-1}}{k} \sum_{\sum_{i=1}^{k} S_{i}=S} \frac{1}{\prod_{i=1}^{k} S_{i} !} \\
& \left(\sum_{\left\{j_{i}\right\}_{i=1}^{u} \in\{1,2, \cdots k\}} \prod_{i=1}^{u}\left(\frac{S_{j_{i}} !}{\left(S_{j_{i}}-\sum_{k=1}^{r} l_{k}^{i} Q^{k}\right) !}+\cdots\right)\right)
\end{aligned}
$$

To this point, we have transformed the problem to a clear combinatoric one. What we need to do is to reduce the above expression via combinatoric techniques to $K+O\left(\frac{1}{m}\right)$. We can either tackle the combinatoric problem head on, or try to get around it in some way. In the next section, we will see 
how much we can do with pure combinatoric. Then in the further section, we will present a rather simple geometric proof.

\section{Combinatoric Approach.}

To proceed further, we need some more sophisticated combinatoric equalities. Recall from (4.1), we have

$$
\sum_{u=1}^{\infty}(-1)^{u} \sum_{\sum_{j=1}^{u}} \sum_{L^{j}=L} \frac{1}{\prod_{j=1}^{u}\left(L^{j}\right) !}=\frac{(-1)^{l}}{L !}
$$

Use a similar method we can get the following.

Lemma 5.1. For $L \in \mathbf{Z}_{+}^{\mathbf{n}}$ we have

$$
\sum_{u=1}^{\infty} \frac{(-1)^{u-1}}{u} \sum_{\sum_{j=1}^{u} L^{j}=L} \frac{1}{\prod_{j=1}^{u}\left(L^{j}\right) !}= \begin{cases}0, & l>1 \\ 1, & l=1\end{cases}
$$

Proof. Let

$$
I_{L}=\sum_{u=1}^{\infty} \frac{(-1)^{u-1}}{u} \sum_{\sum_{j=1}^{u} L^{j}=L} \frac{1}{\prod_{j=1}^{u}\left(L^{j}\right) !}
$$

Notice that

$$
\sum_{L} I_{L} T^{L}=\sum_{u=1}^{\infty} \frac{(-1)^{u-1}}{u}\left(e^{t}-1\right)^{u}=t
$$

The equation in the lemma then follow.

With the above two equalities in mind, we will show some generalizations of them. The method is similar, but need more care. The following elementary equality is used in the proofs.

$$
(1+x)^{-r}=\sum_{u}^{\infty}(-1)^{u+r-1} \frac{(u+r-1) !}{u !(r-1) !} x^{u}
$$


Lemma 5.2. For $L, A_{1}, A_{2}, \cdots A_{r} \in \mathbf{Z}_{+}^{\mathbf{n}}$ and $A=\sum_{i=1}^{r} A_{i}$ we have

$$
\begin{gathered}
\sum_{u=r}^{\infty}(-1)^{u} \sum_{\sum_{j=1}^{u} L_{L^{j}}=L} \frac{1}{\prod_{j=1}^{u}\left(L^{j}\right) !}\left(\sum_{\substack{j_{k}=1 \\
k=1,2, \cdots r}}^{u} \prod_{k=1}^{r} \frac{L^{j_{k} !}}{\left(L^{j_{k}}-A_{k}\right) !}\right) \\
=\left\{\begin{array}{cl}
(-1)^{r+l-a} \frac{r !}{(L-A) !}, & L \geq A \\
0, & L<A
\end{array}\right.
\end{gathered}
$$

Proof. Let

$$
g(L, u)=\sum_{\sum_{j=1}^{u} L^{j}=L} \frac{1}{\prod_{j=1}^{u}\left(L^{j}\right) !}
$$

Then

$$
\begin{aligned}
\sum_{L} t^{L} \sum_{u=r}^{\infty}(-1)^{u} \sum_{\sum_{j=1}^{u} L^{j}=L} \frac{1}{\prod_{j=1}^{u}\left(L^{j}\right) !}\left(\begin{array}{c}
\sum_{j_{k}=1}^{u} \prod_{k=1}^{r} \frac{L^{j_{k}} !}{\left(L^{j_{k}}-A_{k}\right) !} \\
k=1,2, \cdots r
\end{array}\right) \\
\quad=\sum_{L} t^{L} \sum_{u=r}^{\infty}(-1)^{u} \frac{u !}{(u-r) !} \sum_{i=0}^{r}\left(\begin{array}{c}
r \\
i
\end{array}\right) g(L-A, u-i) \\
\quad=\sum_{u}^{\infty}(-1)^{u} \frac{u !}{(u-r) !} \sum_{i=0}^{r}\left(\begin{array}{c}
r \\
i
\end{array}\right) \sum_{L} t^{L} g(L-A, u-i) \\
=\sum_{u}^{\infty}(-1)^{u} \frac{u !}{(u-r) !} t^{A} \sum_{i=0}^{r}\left(\begin{array}{c}
r \\
i
\end{array}\right)\left(e^{t}-1\right)^{u-i} \\
=t^{A} e^{t r} \sum_{u}^{\infty}(-1)^{u} \frac{u !}{(u-r) !}\left(e^{t}-1\right)^{u-r} \\
=(-1)^{r} r ! e^{-t} t^{A}
\end{aligned}
$$

Compare the coefficients, we get the lemma.

Lemma 5.3. For $L, A_{1}, A_{2}, \cdots A_{r} \in \mathbf{Z}_{+}^{\mathbf{n}}$ and $A=\sum_{i=1}^{r} A_{i}$ we have 
(i) when $r>0$

$$
\begin{aligned}
\sum_{u=r}^{\infty} \frac{(-1)^{u-1}}{u} \sum_{\sum_{j=1}^{u} L^{j}=L} \frac{1}{\prod_{j=1}^{u}\left(L^{j}\right) !} & \left(\begin{array}{cc}
\sum_{j_{k}=1}^{u} \prod_{k=1}^{r} \frac{L^{j_{k}} !}{\left(L^{j_{k}}-A_{k}\right) !} \\
k=1,2, \cdots r
\end{array}\right) \\
& =\left\{\begin{array}{cc}
(-1)^{r-1}(r-1) !, & L=A \\
0, & L \neq A
\end{array}\right.
\end{aligned}
$$

(ii) when $r=0$

$$
\sum_{u=1}^{\infty} \frac{(-1)^{u-1}}{u} \sum_{\sum_{j=1}^{u} L^{j}=L} \frac{1}{\prod_{j=1}^{u}\left(L^{j}\right) !}= \begin{cases}1, & l=1 \\ 0, & l \neq 1\end{cases}
$$

Proof. Case $(i i)$ is simple, we will prove case $(i)$, so assume $r>0$.

$$
\begin{aligned}
\sum_{L} t^{L} \sum_{u=r}^{\infty} \frac{(-1)^{u-1}}{u} \sum_{\sum_{j=1}^{u} L^{j}=L} \frac{1}{\prod_{j=1}^{u}\left(L^{j}\right) !} \\
\left(\begin{array}{c}
\sum_{j_{k}=1}^{u} \prod_{k=1}^{r} \frac{L^{j_{k} !}}{\left(L^{j_{k}}-A_{k}\right) !} \\
k=1,2, \cdots r
\end{array}\right) \\
=\sum_{L} t^{L} \sum_{u=r}^{\infty} \frac{(-1)^{u-1}}{u} \frac{u !}{(u-r) !} \sum_{i=0}^{r}\left(\begin{array}{c}
r \\
i
\end{array}\right) g(L-A, u-i) \\
=\sum_{u}^{\infty} \frac{(-1)^{u-1}}{u} \frac{u !}{(u-r) !} \sum_{i=0}^{r}\left(\begin{array}{c}
r \\
i
\end{array}\right) \sum_{L} t^{L} g(L-A, u-i) \\
=\sum_{u}^{\infty} \frac{(-1)^{u-1}}{u} \frac{u !}{(u-r) !} t^{A} \sum_{i=0}^{r}\left(\begin{array}{c}
r \\
i
\end{array}\right)\left(e^{t}-1\right)^{u-i} \\
=t^{A} e^{t r} \sum_{u}^{\infty}(-1)^{u-1} \frac{(u-1) !}{(u-r) !}\left(e^{t}-1\right)^{u-r}
\end{aligned}
$$




$$
\begin{aligned}
& =(r-1) ! t^{A} e^{t r} \sum_{u}^{\infty}(-1)^{u+r-1} \frac{(u+r-1) !}{u !(r-1) !}\left(e^{t}-1\right)^{u} \\
& =(-1)^{r-1}(r-1) ! t^{A} e^{t r}\left(\frac{1}{e^{t}}\right)^{r} \\
& =(-1)^{r-1}(r-1) ! t^{A}
\end{aligned}
$$

Compare the coefficients, we get the lemma.

In our proof, we will also need the following type of equality. The idea is to express polynomials of $s$ in term of polynomials like: $\frac{s !}{(s-m) !}$ for $m \in \mathbf{Z}_{+}$.

\section{Lemma 5.4.}

$$
\frac{(s+p) !}{(s+p-q) !}=\sum_{i=0}\left(\begin{array}{c}
p \\
i
\end{array}\right)\left(\begin{array}{c}
q \\
i
\end{array}\right) \frac{i ! s !}{(s-q+i) !}
$$

Proof. It is easy to show by induction.

We have the following multivariable version of this lemma, notice that the multi-index $I \in \mathbf{Z}_{+}^{\mathbf{n}}$.

\section{Lemma 5.5.}

$$
\frac{(S+P) !}{(S+P-Q) !}=\sum_{I}\left(\begin{array}{c}
P \\
I
\end{array}\right)\left(\begin{array}{c}
Q \\
I
\end{array}\right) \frac{I ! S !}{(S-Q+I) !}
$$

Proof. This is a corollary of previous lemma, if one notice that the left hand side of the equation in this lemma is a product with each factor being of the shape of the left hand side of the equation of the previous lemma, and the right hand side is expanded form of a product.

We are actually interested in

$$
\prod_{j=1}^{u} \frac{\left(S+\sum_{i=1}^{j}\left(P_{i}-Q_{i}\right)+Q_{j}\right) !}{\left(S+\sum_{i=1}^{j}\left(P_{i}-Q_{i}\right)\right) !} .
$$

It turns out that we can handle this too. 
Lemma 5.6.

$$
\begin{aligned}
& \prod_{j=1}^{u} \frac{\left(S+\sum_{i=1}^{j}\left(P_{i}-Q_{i}\right)+Q_{j}\right) !}{\left(S+\sum_{i=1}^{j}\left(P_{i}-Q_{i}\right)\right) !} \\
&=\sum_{I} B_{I}(L, P, Q) \frac{S !}{\left(S-\left(\sum_{j=1}^{u} Q_{j}\right)+I\right) !}
\end{aligned}
$$

Here

$$
B_{I}(L, P, Q)=\sum_{\sum_{k=1}^{u} I_{k}=I}\left(\prod_{j=1}^{u}\left(\begin{array}{c}
P_{j} \\
I_{j}
\end{array}\right)\left(\begin{array}{c}
\sum_{m=j}^{u}\left(Q_{m}-I_{m}\right)+I_{j} \\
I_{j}
\end{array}\right) I_{j} !\right)
$$

Proof. It is straightforward by suitable induction using previous lemma.

Add in the influence of $\operatorname{det} g$, we get

$$
\begin{aligned}
& \left(\prod_{j=1}^{u} \frac{\left(S+\sum_{i=1}^{j}\left(P_{i}-Q_{i}\right)+Q_{j}\right) !}{\left(S+\sum_{i=1}^{j}\left(P_{i}-Q_{i}\right)\right) !}\right)\left(\prod_{j=1}^{u} \sum_{\substack{I_{j} \subset(1,2, \cdots, r) \\
K_{j} \subset(1,2, \cdots, n)}}\right. \\
& \left.\operatorname{det}\left(P_{, K_{j}}^{I_{j}}\right) \operatorname{det}\left(Q_{, K_{j}}^{I_{j}}\right)\left(L^{j}\right)^{e_{I_{j}}} \frac{(-1)^{\left|K_{j}\right|}}{\prod_{k \in K_{j}}\left(s_{, k}+\sum_{i=1}^{j}\left(p_{i, k}-q_{i, k}\right)+q_{j, k}\right)}\right) \\
& =\sum_{I_{j} \subset(1,2, \cdots, r)}\left(\prod_{j=1}^{u} \operatorname{det}\left(P_{, K_{j}}^{I_{j}}\right) \operatorname{det}\left(Q_{, K_{j}}^{I_{j}}\right)\left(L^{j}\right)^{e_{I_{j}}}(-1)^{\left|K_{j}\right|}\right) \\
& K_{j} \subset(1,2, \cdots, n) \\
& 1 \leq j \leq u \\
& \left(\prod_{j=1}^{u} \frac{\left(S+\sum_{i=1}^{j}\left(P_{i}-Q_{i}\right)+Q_{j}-e_{K_{j}}\right) !}{\left(S+\sum_{i=1}^{j}\left(P_{i}-Q_{i}\right)\right) !}\right) \\
& =\sum_{I_{j} \subset(1,2, \cdots, r)}\left(\prod_{j=1}^{u} \operatorname{det}\left(P_{, K_{j}}^{I_{j}}\right) \operatorname{det}\left(Q_{, K_{j}}^{I_{j}}\right)\left(L^{j}\right)^{e_{I_{j}}}(-1)^{\left|K_{j}\right|}\right) \\
& K_{j} \subset(1,2, \cdots, n) \\
& 1 \leq j \leq u
\end{aligned}
$$




$$
\begin{aligned}
& \left(\sum_{A} B_{A}\left(\left\{P_{j}-K_{j}\right\}_{j=1}^{u},\left\{Q_{j}-K_{j}\right\}_{j=1}^{u}\right) \frac{S !}{\left(S-\left(\sum_{j=1}^{u} Q_{j}\right)+K+A\right) !}\right) \\
= & \sum_{A} \frac{S !}{\left(S-\left(\sum_{j=1}^{u} Q_{j}\right)+A\right) !} \\
& \sum^{I_{j} \subset(1,2, \cdots, r)} \\
& K_{j} \subset(1,2, \cdots, n) \\
& \quad B_{A-K}\left(\left\{P_{j}-K_{j}\right\}_{j=1}^{u},\left\{Q_{j}-K_{j}\right\}_{j=1}^{u}\right) \\
& \left(\prod_{j=1}^{u} \operatorname{det}\left(P_{, K_{j}}^{I_{j}}\right) \operatorname{det}\left(Q_{, K_{j}}^{I_{j}}\right)\left(L^{j}\right)^{e_{I_{j}}}(-1)^{\left|K_{j}\right|}\right) \\
= & \sum_{A} \hat{B}_{A}(L, P, Q) \frac{S !}{\left(S-\left(\sum_{j=1}^{u} Q_{j}\right)+A\right) !}
\end{aligned}
$$

\section{Therefore}

$$
\begin{aligned}
\hat{B}_{A}(L, P, Q)= & \sum_{\substack{I_{j} \subset(1,2, \cdots, r) \\
K_{j} \subset(1,2, \cdots, n) \\
1 \leq j \leq u}} B_{A-K}\left(\left\{P_{j}-K_{j}\right\}_{j=1}^{u},\left\{Q_{j}-K_{j}\right\}_{j=1}^{u}\right) \\
& \left(\prod_{j=1}^{u} \operatorname{det}\left(P_{, K_{j}}^{I_{j}}\right) \operatorname{det}\left(Q_{, K_{j}}^{I_{j}}\right)\left(L^{j}\right)^{e_{I_{j}}}(-1)^{\left|K_{j}\right|}\right)
\end{aligned}
$$

The relation of $\hat{B}_{I}(L, P, Q)$ and $D_{I}(L, P, Q)$ is as following

$$
D_{I}(L, P, Q)=(-1)^{l} L ! \sum_{u=1}^{\infty}(-1)^{u} \sum_{\sum_{i=1}^{u} L^{i}=L} \frac{1}{\prod_{i=1}^{u}\left(L^{i}\right) !} \hat{B}_{I}(L, P, Q)
$$


Notice that the first few terms of right hand side can be written as

$$
\begin{aligned}
& \prod_{j=1}^{u} \frac{\left(S+\sum_{i=1}^{j}\left(P_{i}-Q_{i}\right)+Q_{j}\right) !}{\left(S+\sum_{i=1}^{j}\left(P_{i}-Q_{i}\right)\right) !} \\
& =\frac{S !}{\left(S-\sum_{j=1}^{u} Q_{j}\right) !}+\sum_{i=1}^{r} \sum_{j=1}^{u}\left(P_{j, i} \sum_{m=j}^{u} Q_{m, i}\right) \frac{S !}{\left(S-\sum_{j=1}^{u} Q_{j}+e_{i}\right) !}+\cdots \\
& =\frac{S !}{\left(S-\sum_{i=1}^{r} l_{i} Q^{i}\right) !}+\sum_{j=1}^{r} \hat{B}_{1, j}(L, P, Q) \frac{S !}{\left(S-\sum_{i=1}^{r} l_{i} Q^{i}+e_{j}\right) !}+\cdots
\end{aligned}
$$

Here $\hat{B}_{1, j}(L, P, Q)$ is the same as $\hat{B}_{e_{j}}(L, P, Q)$. From this one can conclude that

Proposition 5.1.

$$
\operatorname{deg}_{L}\left(D_{A}(L, P, Q)\right)=2 a
$$

Proof. Since

$$
B_{A}(L, P, Q)=\sum_{\sum_{k=1}^{u} I_{k}=A}\left(\prod_{j=1}^{u}\left(\begin{array}{c}
P_{j} \\
I_{j}
\end{array}\right)\left(\begin{array}{c}
\sum_{m=j}^{u}\left(Q_{m}-I_{m}\right)+I_{j} \\
I_{j}
\end{array}\right) I_{j} !\right)
$$

Also

$$
\operatorname{deg}_{L}\left(P_{j}\right)=\operatorname{deg}_{L}\left(\sum_{m=j}^{u}\left(Q_{m}-I_{m}\right)+I_{j}\right)=1
$$

and

$$
\operatorname{deg}_{L}\left(\begin{array}{c}
P_{j} \\
I_{j}
\end{array}\right)=\operatorname{deg}_{L}\left(\begin{array}{c}
\sum_{m=j}^{u}\left(Q_{m}-I_{m}\right)+I_{j} \\
I_{j}
\end{array}\right)=\left|I_{j}\right|
$$

We have

$$
\operatorname{deg}_{L}\left(B_{A}(L, P, Q)\right)=2 a
$$

Formula (5.1) gives also

$$
\operatorname{deg}_{L}\left(\hat{B}_{A}(L, P, Q)\right)=2 a
$$

Then by the expression:

$$
D_{A}(L, P, Q)=(-1)^{l} L ! \sum_{u=1}^{\infty}(-1)^{u} \sum_{\sum_{i=1}^{u} L^{i}=L} \frac{1}{\prod_{i=1}^{u}\left(L^{i}\right) !} \hat{B}_{A}(L, P, Q)
$$


and lemma 5.2, we get

$$
\operatorname{deg}_{L}\left(D_{A}(L, P, Q)\right)=2 a
$$

Put above into the expression of $K_{m}$ as in the last equation of formula (4.2) and use lemma 5.3 for variable $S$, we get

$$
\begin{aligned}
& K_{m}=\frac{1}{m} \sum_{S-T} \sum_{L=\left(l_{1}, l_{2}, \cdots l_{r}\right) \neq 0} \prod_{i=1}^{r}\left(-b_{P^{i} \bar{Q}^{i}}\right)^{l_{i}} \sum_{u=1}^{\infty} \\
& \sum_{i=1}^{r} l_{i}\left(P^{i}-Q^{i}\right)=T-S \\
& \sum_{\sum_{i=1}^{u} L^{i}=L} \frac{1}{\prod_{i=1}^{u}\left(L^{i}\right) !}(\sqrt{m} z)^{(T-S)} \\
& \frac{(-1)^{u-1}}{u} \prod_{j=1}^{u}\left(\sum_{I} D_{I}\left(L^{j}, P, Q\right)(\sqrt{m} z)^{\sum_{k=1}^{r} l_{k}^{j} Q^{k}-I}(\sqrt{m} \bar{z})^{\sum_{k=1}^{r} l_{k}^{j} Q^{k}-I}\right) \\
& +\frac{1}{m} \sum_{S}(\sqrt{m} z)^{S}(\sqrt{m} \bar{z})^{S} \sum_{k=1}^{\infty} \frac{(-1)^{k-1}}{k} \sum_{\sum_{i=1}^{k} S_{i}=S} \frac{1}{\prod_{i=1}^{k} S_{i} !} \\
& =\frac{1}{m} \sum_{S-T} \sum_{L=\left(l_{1}, l_{2}, \cdots l_{r}\right) \neq 0} \prod_{i=1}^{r}\left(-b_{P^{i} \bar{Q}^{i}}\right)^{l_{i}} \\
& \sum_{i=1}^{r} l_{i}\left(P^{i}-Q^{i}\right)=T-S \\
& \sum_{u=1}^{\infty} \sum_{\sum_{i=1}^{u} L^{i}=L} \frac{1}{\prod_{i=1}^{u}\left(L^{i}\right) !} \\
& \frac{(-1)^{u-1}}{u} \prod_{j=1}^{u}\left(\sum_{I} D_{I}\left(L^{j}, P, Q\right)(\sqrt{m} z)^{\sum_{k=1}^{r} l_{k}^{j} P^{k}-I}(\sqrt{m} \bar{z})^{\sum_{k=1}^{r} l_{k}^{j} Q^{k}-I}\right) \\
& +\frac{1}{m}\left(m|z|^{2}\right) \\
& =\frac{1}{m} \sum_{S-T} \sum_{L=\left(l_{1}, l_{2}, \cdots l_{r}\right) \neq 0} \prod_{i=1}^{r}\left(-b_{P^{i} \bar{Q}^{i}}(\sqrt{m} z)^{P^{i}}(\sqrt{m} \bar{z})^{Q^{i}}\right)^{l_{i}} \\
& \sum_{i=1}^{r} l_{i}\left(P^{i}-Q^{i}\right)=T-S \\
& \sum_{u=1}^{\infty} \frac{(-1)^{u-1}}{u} \sum_{\sum_{i=1}^{u} L^{i}=L} \frac{1}{\prod_{i=1}^{u}\left(L^{i}\right) !}
\end{aligned}
$$




$$
\begin{aligned}
& \prod_{j=1}^{u}\left(\sum_{I} D_{I}\left(L^{j}, P, Q\right)(\sqrt{m} z)^{-I}(\sqrt{m} \bar{z})^{-I}\right)+|z|^{2} \\
= & \frac{1}{m} \sum_{L \neq 0} \prod_{i=1}^{r}\left(-b_{P^{i} \bar{Q}^{i}}(\sqrt{m} z)^{P^{i}}(\sqrt{m} \bar{z})^{Q^{i}}\right)^{l_{i}} \\
& \sum_{u=1}^{\infty} \frac{(-1)^{u-1}}{u} \sum_{\sum_{i=1}^{u} L^{i}=L} \frac{1}{\prod_{i=1}^{u}\left(L^{i}\right) !} \\
& \sum_{I}\left(\sum_{\sum_{j=1}^{u} I^{j}=I^{j=1}}^{u} \prod_{I^{j}}\left(L^{j}, P, Q\right)\right)(\sqrt{m} z)^{-I}(\sqrt{m} \bar{z})^{-I}+|z|^{2} \\
= & |z|^{2}+\frac{1}{m} \sum_{I}(\sqrt{m} z)^{-I}(\sqrt{m} \bar{z})^{-I} \\
& \left(\sum_{L \neq 0} X^{L} \sum_{u=1}^{\infty} \frac{(-1)^{u-1}}{u} \sum_{\sum_{i=1}^{u} L^{i}=L} \frac{1}{\prod_{i=1}^{u}\left(L^{i}\right) !} E_{I}(L, P, Q)\right)
\end{aligned}
$$

Where $X=\left(x^{1}, x^{2}, \cdots x^{r}\right), x^{i}=-b_{P^{i} \bar{Q}^{i}}(\sqrt{m} z)^{P^{i}}(\sqrt{m} \bar{z})^{Q^{i}}$, and

$$
\left.E_{I}(L, P, Q)=\sum_{\sum_{j=1}^{u} I^{j}=I^{j=1}} \prod_{I^{j}}^{u} L^{j}, P, Q\right)
$$

Recall again that what we want to show is that all the $m^{r}$ terms with $r>0$ in the expansion of $K_{m}(z, \bar{z})$ vanish and the terms that do not involve $m$ should equal to $K(z, \bar{z})$. It is not hard to see with the help of lemma 5.3 ( $i i)$ and from the fact $E_{0}(L, P, Q)=1$ that the terms related to $I=0$ in $K_{m}$ give exactly the expansion of $K(z, \bar{z})$. Then what we expect clearly is that the $I>0$ terms only involve negative $m$-power. More precisely we have $K_{m}=K_{m}^{0}+K_{m}^{\prime}$

$$
\begin{aligned}
= & \left(|z|^{2}+\frac{1}{m} \sum_{L} X^{L} \sum_{u=1}^{\infty} \frac{(-1)^{u-1}}{u} \sum_{\sum_{i=1}^{u} L^{i}=L} \frac{1}{\prod_{i=1}^{u}\left(L^{i}\right) !} E_{0}(L, P, Q)\right) \\
& +\frac{1}{m} \sum_{I \neq 0}(\sqrt{m} z)^{-I}(\sqrt{m} \bar{z})^{-I}
\end{aligned}
$$




$$
\left(\sum_{L} X^{L} \sum_{u=1}^{\infty} \frac{(-1)^{u-1}}{u} \sum_{\sum_{i=1}^{u} L^{i}=L} \frac{1}{\prod_{i=1}^{u}\left(L^{i}\right) !} E_{I}(L, P, Q)\right)
$$

\section{Proposition 5.2.}

$K_{m}^{0}=|z|^{2}+\frac{1}{m} \sum_{L} X^{L} \sum_{u=1}^{\infty} \frac{(-1)^{u-1}}{u} \sum_{\sum_{i=1}^{u} L^{i}=L} \frac{1}{\prod_{i=1}^{u}\left(L^{i}\right) !} E_{0}(L, P, Q)=K$

Proof. Clearly

$$
\begin{aligned}
E_{0}(L, P, Q) & =D_{0}(L, P, Q) \\
& =(-1)^{l} L ! \sum_{u=1}^{\infty}(-1)^{u} \sum_{\sum_{i=1}^{u} L^{i}=L} \frac{1}{\prod_{i=1}^{u}\left(L^{i}\right) !} \hat{B}_{0}(L, P, Q)
\end{aligned}
$$

By the obvious fact $\hat{B}_{0}(L, P, Q)=B_{0}(L, P, Q)=1$ and formula (4.1), we have

$$
E_{0}(L, P, Q)=1
$$

This together with lemma 5.3(ii) give us

$$
\begin{aligned}
K_{m}^{0} & =|z|^{2}+\frac{1}{m} \sum_{L} X^{L} \sum_{u=1}^{\infty} \frac{(-1)^{u-1}}{u} \sum_{\sum_{i=1}^{u} L^{i}=L} \frac{1}{\prod_{i=1}^{u}\left(L^{i}\right) !} \\
& =|z|^{2}+\frac{1}{m} \sum_{i=1}^{r} x^{i} \\
& =|z|^{2}+\frac{1}{m} \sum_{i=1}^{r}\left(-b_{P^{i} \bar{Q}^{i}}(\sqrt{m} z)^{P^{i}}(\sqrt{m} \bar{z})^{Q^{i}}\right) \\
& =|z|^{2}+\sum_{i=1}^{r} c_{P^{i} \bar{Q}^{i}} z^{P^{i}} \bar{z}^{Q^{i}}=K
\end{aligned}
$$

It is instructive to analyze the weight of $m$ in various terms. From the definition, one can easily see that $x^{i}$ all have weight 1 , also notice that $(\sqrt{m} z)^{-I}(\sqrt{m} \bar{z})^{-I}$ has weight $-i$, we should expect 


$$
F_{I}(X, P, Q)=\sum_{L} X^{L} \sum_{u=1}^{\infty} \frac{(-1)^{u-1}}{u} \sum_{\sum_{i=1}^{u} L^{i}=L} \frac{1}{\prod_{i=1}^{u}\left(L^{i}\right) !} E_{I}(L, P, Q)
$$

being a polynomial of degree less than or equal to $i$. It is easy to deduce from (5.1) that $\operatorname{deg}_{L}\left(E_{I}(L, P, Q)\right)=2 i$, which guarantee that $\operatorname{deg}_{X}\left(F_{I}(X, P, Q)\right)=2 i$ with the help of lemma 5.3. But this falls short to satisfy our need, which requires vanishing of all coefficients of degree larger than $i$. We do not know a coherent way to show the vanishing to all order. But apparently, there is an algorithm to check the vanishing. For any finite order, there only involve finite steps of checking. Although it could be tedious, in principle, it works for checking convergence up to any finite order. Before proceed further, we would like to examine first that what implication we would have if we have already proved $\operatorname{deg}_{X}\left(F_{J}(X, P, Q)\right) \leq j$ for $j \leq i$.

Proposition 5.3. Assume that $\operatorname{deg}_{X}\left(F_{J}(X, P, Q)\right) \leq j$ for $j \leq i$. Then we have

$$
\left\|g_{m}-g\right\|_{C^{2 i+3}}=O\left(\frac{1}{m}\right)
$$

or more precisely

$$
\left\|g_{m}-g\right\|_{C^{a, b}}=O\left(\frac{1}{m}\right) \quad \text { for } a \leq i+1 \text { or } b \leq i+1
$$

Proof. Recall from formula (5.4) and Proposition 5.2 that

$$
K_{m}=K+\frac{1}{m} \sum_{I \neq 0}(\sqrt{m} z)^{-I}(\sqrt{m} \bar{z})^{-I} F_{I}(X, P, Q)
$$

Since in term of $m, X$ has weight one. In order for a term involving $X^{L}$ in $F_{J}(X, P, Q)$ to have non-negative $m$-power contribution, $l$ has to be bigger than $i$. Its $z$ degree will be $(P \cdot L-j, Q \cdot L-j)$. Our assumptions clearly imply that $F_{J}(X, P, Q)$ will not contribute non-negative $m$ power if $j \leq i$. Only terms of $F_{J}(X, P, Q)$ involving $X^{L}$ for $l>j>i$ will matter. Recall also that for $c_{P \bar{Q}}$ we always have $(p, q) \geq(2,2)$ (with the first term being the curvature). So the corresponding $z$ degree for those terms are

$$
(P \cdot L-j, Q \cdot L-j) \geq(2 l-j, 2 l-j) \geq(i+3, i+3)
$$


These imply the $C^{a, b}$ convergence of Kähler potential for $a \leq i+2$ or $b \leq i+2$, which in turn imply our proposition.

Especially, for the case $i=0$, namely without any checking, we already have

\section{Corollary 5.1.}

$$
\left\|g_{m}-g\right\|_{C^{3}}=O\left(\frac{1}{m}\right)
$$

i.e. induced Bergmann metrics are $C^{3}$ convergent to the original metric.

Remark 5.1. Our previous combinatoric arguments were quite complicated, because we were aiming at the $C^{\infty}$ convergence. If we only care about $C^{3}$ convergence, then use our idea, it is not hard to write a simple direct proof of the above result. The key point is that we do not need to check any combinatoric identities that will have to be checked for higher order convergence.

As an exercise, we will check the case $i=1$.

Recall that

$$
\begin{aligned}
F_{e_{j}}(X, P, Q) & =\sum_{L} X^{L} \sum_{u=1}^{\infty} \frac{(-1)^{u-1}}{u} \sum_{\sum_{i=1}^{u} L^{i}=L} \frac{1}{\prod_{i=1}^{u}\left(L^{i}\right) !} E_{e_{j}}(L, P, Q) \\
E_{e_{j}}(L, P, Q) & =\sum_{k=1}^{u} D_{e_{j}}\left(L^{k}, P, Q\right) \\
D_{e_{j}}(L, P, Q) & =(-1)^{l} L ! \sum_{u=1}^{\infty}(-1)^{u} \sum_{\sum_{i=1}^{u} L^{i}=L} \frac{L !}{\prod_{i=1}^{u}\left(L^{i}\right) !} \hat{B}_{e_{j}}(L, P, Q) \\
B_{e_{j}}(L, P, Q) & =\sum_{k=1}^{u} P_{k, j} \sum_{m=k}^{u} Q_{m, j} \\
& =\sum_{k=1}^{u} \sum_{i_{1}=1}^{r} l_{i_{1}}^{k} P_{, j}^{i_{1}} \sum_{m=k}^{u} \sum_{i_{2}=1}^{r} l_{i_{2}}^{m} Q_{, j}^{i_{2}} \\
& =\sum_{i_{1}, i_{2}=1}^{r} P_{, j}^{i_{1}} Q_{, j}^{i_{2}}\left(\frac{1}{2} \sum_{k \neq m}^{u} \frac{L^{k} !}{\left(L^{k}-e_{i_{1}}\right) !} \frac{L^{m !}}{\left(L^{m}-e_{i_{2}}\right) !}\right.
\end{aligned}
$$




$$
\begin{aligned}
& \left.+\sum_{k=1}^{u} \frac{L^{k} !}{\left(L^{k}-e_{i_{1}}-e_{i_{2}}\right) !}\right) \\
& +\sum_{i=1}^{r} P_{, j}^{i} Q_{, j}^{i} \sum_{k=1}^{u} \frac{L^{k} !}{\left(L^{k}-e_{i}\right) !} \\
\hat{B}_{e_{j}}(L, P, Q)= & B_{e_{j}}(L, P, Q)+\sum_{k=1}^{u} \sum_{i=1}^{r} P_{, j}^{i} Q_{, j}^{i} l_{i}^{k}(-1) \\
= & \sum_{i_{1}, i_{2}=1}^{r} P_{, j}^{i_{1}} Q_{, j}^{i_{2}}\left(\frac{1}{2} \sum_{k \neq m}^{u} \frac{L^{k} !}{\left(L^{k}-e_{i_{1}}\right) !} \frac{L^{m} !}{\left(L^{m}-e_{i_{2}}\right) !}\right. \\
& +\sum_{i=1}^{r} P_{, j}^{i} Q_{, j}^{i} \sum_{k=1}^{u} \frac{L^{k} !}{\left(L^{k}-e_{i}\right) !}-\sum_{k=1}^{u} \sum_{i=1}^{r} P_{, j}^{i} Q_{, j}^{i} l_{i}^{k} \\
= & \sum_{i_{1}, i_{2}=1}^{r} P_{, j}^{i_{1}} Q_{, j}^{i_{2}}\left(\frac{1}{2} \sum_{k \neq m}^{u} \frac{L^{k} !}{\left(L^{k}-e_{i_{1}}\right) !} \frac{L^{m} !}{\left(L^{m}-e_{i_{2}}\right) !}\right. \\
& \left.+\sum_{k=1}^{u} \frac{L^{k} !}{\left(L^{k}-e_{i_{1}}-e_{i_{2}}\right) !}\right)
\end{aligned}
$$

With the help of lemma 5.2, we get

$$
\begin{aligned}
D_{e_{j}}(L, P, Q)= & \sum_{i_{1}, i_{2}=1}^{r} P_{, j}^{i_{1}} Q_{, j}^{i_{2}}\left(\frac{1}{2}(-1)^{l} \frac{2 ! L !}{\left(L-e_{i_{1}}-e_{i_{2}}\right) !}\right. \\
& \left.+(-1)^{l-1} \frac{L !}{\left(L-e_{i_{1}}-e_{i_{2}}\right) !}\right) \\
& +\sum_{i=1}^{r} P_{, j}^{i} Q_{, j}^{i}(-1)^{l} \frac{L !}{\left(L-e_{i}\right) !}-\sum_{i=1}^{r} P_{, j}^{i} Q_{, j}^{i}(-1)^{l} \frac{L !}{\left(L-e_{i}\right) !}=0
\end{aligned}
$$

Which is clearly degree one on $L$. So $i=1$ case checking is confirmed. This checking together with Proposition 5.3 give us

Corollary 5.2.

$$
\left\|g_{m}-g\right\|_{C^{5}}=O\left(\frac{1}{m}\right)
$$

i.e. induced Bergmann metrics are $C^{5}$ convergent to the original metric. 
We also checked the $i=2$ case, which will imply $C^{7}$ convergence. It is not impossible to check a few steps further. But complicity will increase and one has to stop at some point with this method. The geometric proof presented in the next section will imply all the necessary vanishing in this section.

\section{Geometric Approach.}

In this section, we will give a very simple and complete geometric proof of the $C^{\infty}$ convergence of Bergmann metrics, which is qualified of being completely different from the combinatoric one presented in the previous section. Therefore, a by-product is that this result imply those unsolved combinatoric problems at the end of the previous section, which still look sort of mysterious.

Recall that what we want to show is that all the $m^{r}$ terms with $r>0$ in the expansion of $K_{m}(z, \bar{z})$ vanish and the terms that do not involve $m$ should equal to $K(z, \bar{z})$. From the expression of $K_{m}(z, \bar{z})$ in formula (4.2), we can get the following observation, which turns out to be enough to initiate our geometric proof. Consider the expansion of $K_{m}(z, \bar{z})$ as in formula (4.2) with respect to $z$, let $y_{S \bar{T}}$ be the coefficients of $z^{S} \bar{z}^{T}$ term omitting the part that involve negative powers of $m$. Then $y_{S \bar{T}}=f_{S \bar{T}}\left(m, c_{P \bar{Q}}\right)$ is a function of $m$ and some $c_{P \bar{Q}}$.

Proposition 6.1. $y_{S \bar{T}}=f_{S \bar{T}}\left(m, c_{P \bar{Q}}\right)$ is a polynomial of $m$ and $c_{P \bar{Q}}$ for $p+q \leq s+t$ with degree of each variable bounded from above with respect to $S, T$.

Proof. It is clear, if one observe that $z^{S} \bar{z}^{T}$ is accompanied by the factor $m^{\frac{s+t}{2}}$, and all $b_{P \bar{Q}}$ contain a negative $m$-power of $m^{1-\frac{p+q}{2}}$. So $\operatorname{deg}_{m} f_{S \bar{T}} \leq \frac{s+t}{2}$ and $\operatorname{deg}_{b_{P \bar{Q}}}$ large terms will have negative $m$-power and will be omitted.

Let $\left\{f_{m}\right\}_{m=1}^{\infty}$ be a sequence of real functions, such that $\lim _{m \rightarrow \infty} f_{m}=f_{0}$. The idea of our geometric prove can be best illustrated in the following lemma.

Lemma 6.1. For $r \leq 0$, assume that

$$
\lim _{m \rightarrow \infty} f_{m}=f_{0}
$$

and

$$
\lim _{m \rightarrow \infty} m^{r} f_{m}^{\prime}=g
$$


(i) if $r<0$, then $g=0$,

(ii) if $r=0$, then $g=f_{0}^{\prime}$

Proof. Take a test function $\varphi$, then $\lim _{m \rightarrow \infty} m^{r} f_{m}^{\prime}=g$ implies:

$$
\begin{aligned}
\int g \varphi & =\lim _{m \rightarrow \infty} \int m^{r} f_{m}^{\prime} \varphi=\lim _{m \rightarrow \infty}-m^{r} \int f_{m} \varphi^{\prime} \\
& =\lim _{m \rightarrow \infty}\left(-m^{r}\right) \lim _{m \rightarrow \infty} \int f_{m} \varphi^{\prime}=\left(\lim _{m \rightarrow \infty}-m^{r}\right) \int f_{0} \varphi^{\prime} \\
& = \begin{cases}0, & r<0 \\
\int f_{0}^{\prime} \varphi, & r=0\end{cases}
\end{aligned}
$$

Therefore

$$
g=\left\{\begin{array}{cc}
0 & r<0 \\
f_{0}^{\prime} & r=0
\end{array}\right.
$$

For a point $m_{0} \in M$, we would like to discuss the convergence on the canonical coordinate chart: $w=\phi_{m_{0}}: U_{m_{0}} \longrightarrow \mathbf{C}^{\mathbf{n}}$. Recall that for $\phi_{m_{0}}^{-1}(w) \in U_{m_{0}}$, we can choose a smooth family of canonical coordinates

$$
z=\phi_{w}: U_{w} \longrightarrow \mathbf{C}^{\mathbf{n}}
$$

with the convention that $\phi_{0}=\phi_{m_{0}}$, which amounts to pick a smooth section of the principle bundle of orthonormal frames over $U_{m_{0}}$ passing through the canonical frame at $m_{0}$.

To prove the convergence of $\left\{g_{m}\right\}$ to $g$, we will use the induction on the order of convergence. Assume that $\left\{g_{m}\right\}$ converge to $g$ up to $C^{l-1}$, we need to show that for any $L$ with respect to $l(L=(P, Q), p+q=l)$, $\partial_{L} g_{m}$ converge to $\partial_{L} g$. Under coordinate $w$, let $g(w)=g_{i j} d w^{i} d \bar{w}^{j}$, and $g_{m}(w)=g_{m, i j} d w^{i} d \bar{w}^{j}$, written in local coordinate, we need to show that $\partial_{L} g_{m, i j}$ converge to $\partial_{L} g_{i j}$. Notice that

$$
\begin{gathered}
\partial_{L} g_{i j}(w)=\partial_{L+\left(e_{i}, e_{j}\right)} K^{w}(w)=\sum_{M} \frac{\partial z^{M}}{\partial w^{L+\left(e_{i}, e_{j}\right)}} \partial_{M} K^{w}(z)+h_{L} \\
\partial_{L} g_{m, i j}(w)=\partial_{L+\left(e_{i}, e_{j}\right)} K_{m}^{w}(w)=\sum_{M} \frac{\partial z^{M}}{\partial w^{L+\left(e_{i}, e_{j}\right)}} \partial_{M} K_{m}^{w}(z)+h_{m, L}
\end{gathered}
$$

Here $\operatorname{deg} M=(p+1, q+1)$, and $\frac{\partial z^{M}}{\partial w^{L+\left(e_{i}, e_{j}\right)}}$ indicate a sum of fractions with nominators being permutation of factors, which are exactly the factors one 
get for the highest degree derivatives after using chain rule. Notice that at $w=0, \frac{\partial z^{M}}{\partial w^{L+\left(e_{i}, e_{j}\right)}}=\delta_{L+\left(e_{i}, e_{j}\right)}^{M} \cdot h_{L}$ and $h_{m, L}$ indicate the parts that contain derivatives of lower order. by our inductive assumption, $\left\{h_{m, L}\right\}$ converge to $h_{L}$. We only need to deal with the highest order terms. According to Proposition 6.1, the part of $\partial_{L} g_{m, i j}(w)$ that do not involve negative powers of $m$ (denote by $g_{L}\left(w, m, c_{P \bar{Q}}(w)\right)$ ) is a polynomial on $m$ and some $c_{P \bar{Q}}(w)$ of bounded degree. Let $\operatorname{deg}_{m}\left(g_{L}\right)=d_{L}$, then

$$
g_{L}\left(w, m, c_{P \bar{Q}}(w)\right)=\sum_{i=1}^{d_{L}} g_{L, i}(w) m^{i}
$$

we can conclude that

$$
\lim _{m \rightarrow \infty} m^{-d_{L}} \partial_{L} g_{m, i j}(w)=\lim _{m \rightarrow \infty} m^{-d_{L}} g_{L}\left(w, m, c_{P \bar{Q}}(w)\right)=g_{L, d_{L}}(w)
$$

According to lemma 6.1, we have

$$
\lim _{m \rightarrow \infty} m^{-d_{L}} \partial_{L} g_{m, i j}(w)=g_{L, d_{L}}(w)=0
$$

unless $d_{L}=0$. Therefore $\operatorname{deg}_{m}\left(g_{L}\right)=0$ and use induction assumption and lemma 6.1 again, we get

$$
\lim _{m \rightarrow \infty} \partial_{L} g_{m, i j}(w)=\partial_{L} g_{i j}(w)
$$

Now by the induction, we have therefore proved the $C^{\infty}$ convergence of the Bergmann metrics, as stated in the following theorem, modulo the starting point of the induction, the $C^{0}$ convergence of the Bergmann metrics, which we can refer to corollary 5.1 in previous chapter or Tian's proof, although it is not hard to give it a direct proof.

Theorem 6.1. For any $l \in \mathbf{Z}_{+}$

$$
\left\|g_{m}-g\right\|_{C^{l}}=O_{l}\left(\frac{1}{m}\right)
$$

i.e. induced Bergmann metrics are $C^{\infty}$ convergent to the original metric.

The subindex $l$ in the right hand side indicate its dependence on $l$.

A straight forward corollary of this theorem is those combinatoric vanishing problems in the end of the previous section, which we was not able to solve directly. 
Corollary 6.1.

$$
\operatorname{deg}_{X}\left(F_{I}(X, P, Q)\right)=i
$$

Here $F_{I}(X, P, Q)$ is defined in the previous section.

Remark 6.1. Anybody, who have ventured through last section, must have realized how simple the geometric proof is. Here again, we witness the power of analysis. Nevertheless this proof is not as straight forward as the combinatoric one. Does not really help to uncover the combinatoric mystery in the previous section. For this reason, we still like to present the combinatoric approach toward the problem, which seems quite interesting in its own.

\section{Application.}

In this section we will discuss an interesting application of the convergence of Bergmann metric. For $(M, \omega)$ a Kähler manifold with the associated positive Hermitian line bundle $(L, h)$, where $c_{1}(L, h)=\omega$, in arithmetic geometry, one usually need some quantitive result related to the Hermitian metric. A typical result is the following:

Theorem 7.1. For any positive Hermitian line bundle $(L, h)$ and $\epsilon>0$, there exists an $n_{0}$, such that for any $p \in M$ and $n>n_{0}$, there is a section $s \in \Gamma\left(L^{\otimes n}\right)$ such that

$$
\|s\|_{s u p, h} \leq e^{n \epsilon}\|s\|_{h}(p)
$$

It is then interesting to see if the theorem will still be true with $\epsilon=0$. We will show as an application of our techniques and results that this sharp version and some generalization of it is still true.

Theorem 7.2. There exists an $n_{0}$, which depends only on geometry of the positive Hermitian line bundle $(L, h)$, such that for any $p \in M$ and $n>n_{0}$, there is a section $s \in \Gamma\left(L^{\otimes n}\right)$ such that

$$
\|s\|_{s u p, h}=\|s\|_{h}(p)
$$

Proof. For simplicity we will first deal with the case that $L$ is very ample. For a point $p \in M$, we will construct a section of $L^{m}$, for which $\|s\|_{h}$ achieves its maxima at $p$. (Actually we will show that $p$ is the only maximal point.) 
Let $z=\left(z^{1}, z^{2}, \cdots, z^{n}\right)$ be the local K-coordinate of $(M, g)$ with respect to $p$. we will prove the following three statements which together will imply the theorem.

(i) For certain big $C_{2}>0$ and $|z| \geq \frac{C_{2}}{m}$, when $m$ is large enough we have

$$
\|s\|_{h}(0)>\|s\|_{h}(z)
$$

(ii) For certain small $C_{1}>0$ and $|z| \leq \frac{C_{1}}{m}$, when $m$ is large enough we have

$$
\|s\|_{h}(0)>\|s\|_{h}(z)
$$

(iii) For any $C_{2}>C_{1}>0$ and $\frac{C_{1}}{m} \leq|z| \leq \frac{C_{2}}{m}$, when $m$ is large enough we have

$$
\|s\|_{h}(0)>\|s\|_{h}(z)
$$

Since we assume that $L$ is very ample, we have an embedding:

$$
i: M \longrightarrow \mathbf{C P}^{l}
$$

Where $\mathbf{C P}^{l}$ has coordinate $W=\left[W_{0}, W_{1}, \cdots, W_{l}\right], i^{*} O(1)=L . W_{i}$ can be viewed as sections of $O(1)$, we will also use them to denote pull back sections of $L$. We assume that $i(p)=[1,0, \cdots, 0]$.

An important observation is that for $O(1)$ on $\mathbf{C P}^{l}$, it is very easy to find "peak" section needed. $W_{0}$ will work. For $O(m)$, we may take $\left(W_{0}\right)^{m}$. One would hope that $\left(W_{0}\right)^{m}$ will also work for $L^{m}$. But $g$ is not the pull back of the Fubini-Study metric. We need to modify it a little. The key observation here is that the term that spoil the "peak" property is the first order term. So we will need to adjust by some first order term. have

Choose $W$ suitably such that for $w=\left(w^{1}, \cdots, w^{n}\right)$, where $w^{i}=\frac{W_{i}}{W_{0}}$, we

$$
\left.\frac{\partial w}{\partial z}\right|_{z=0}=i d
$$

Also let $h_{0}$ denotes the Hermitian metric on $L=i^{*} O(1)$ induced from usual Hermitian metric on $O(1)$. For peak section $e_{p}$ of $(L, h)$ at point $p \in M$, we have

$$
\left\|e_{p}^{m}\right\|_{h}=e^{-m K}, \text { and }\left\|e_{p}^{m}\right\|_{h_{0}}=1+\sum_{S, T} \frac{G^{T, \bar{S}}}{G^{0, \overline{0}}} z^{S} \bar{z}^{T}
$$

Then for any section $s$ of $L^{m}$, we have

$$
\|s\|_{h}=f(z)\|s\|_{h_{0}}
$$


where

$$
f(z)=\frac{e^{-m K}}{1+\sum_{S, T} \frac{G^{T, \bar{S}}}{G^{0,0}} z^{S} \bar{z}^{T}}=e^{-m\left(K-K_{m}\right)}
$$

By our theorem $\left|K-K_{m}\right|=O\left(\frac{1}{m}\right)$, so we have

$$
f(z)=1-\sum_{i}\left(\frac{G^{0, \overline{e_{i}}}}{G^{0, \overline{0}}} z^{i}+\frac{G^{e_{i}, \overline{0}}}{G^{0, \overline{0}}} \bar{z}^{i}\right)+\sum_{S, T} f_{S, \bar{T}} z^{S} \bar{z}^{T}
$$

where $f_{S, \bar{T}}=O(1)$ and $s+t \geq 2$. An important fact is

$$
G^{0, \overline{e_{i}}}=O\left(\frac{1}{m}\right)
$$

Although apriorily we only have $G^{0, \overline{e_{i}}}=O(1)$. Recall the formula

$$
G^{0, \overline{e_{i}}}=m^{\frac{1}{2}} \sum_{\substack{L=\left(l_{1}, l_{2}, \cdots l_{r}\right) \\ \sum_{i=1}^{r} l_{i}\left(P^{i}-Q^{i}\right)=e_{i}}} \frac{\prod_{i=1}^{r}\left(-b_{P^{i} \bar{Q}^{i}}\right)^{l_{i}}}{L !} F(L, P, Q)
$$

also that

$$
b_{P \bar{Q}}=-c_{P \bar{Q}} m^{1-\frac{p+q}{2}}
$$

Notice that $c_{P \bar{Q}}$ do not depend on $m$ and $p, q \geq 2$, so all the terms of $G^{0, \bar{e}_{i}}$ will be of order at least $O\left(\frac{1}{m}\right)$ unless $L=e_{j}$ and $p^{j}=q^{j}=2$ which is impossible to satisfy $l_{i}\left(P^{i}-Q^{i}\right)=e_{i}$. So we have that estimate (7.1) is correct. Let

$$
s_{0}=\left(W_{0}\right)^{m}, s_{1}=\left(\sum_{i} \frac{G^{0, \overline{e_{i}}}}{G^{0, \overline{0}}} W_{i}\right)\left(W_{0}\right)^{m-1} \text { and } s=s_{0}+s_{1}
$$

Since

$$
\left|K-K_{m}\right|=O\left(\frac{1}{m}\right) \text { and } e^{-m\left(K-K_{m}\right)}=O(1)
$$

We always have that

$$
\|s\|_{h} \leq C\|s\|_{h_{0}}
$$

In case (i) we have

$$
\|s\|_{h}(0)=\left\|s_{0}\right\|_{h}(0)=\left\|s_{0}\right\|_{h_{0}}(0)=1
$$


also

$$
\begin{aligned}
\|s\|_{h}(z) & \leq\left\|s_{0}\right\|_{h}(z)+\left\|s_{1}\right\|_{h}(z) \\
& \leq C\left(\left\|s_{0}\right\|_{h_{0}}(z)+\left\|s_{1}\right\|_{h_{0}}(z)\right)
\end{aligned}
$$

By estimate (7.1) we have

$$
\left\|s_{1}\right\|_{h_{0}}(z)=O\left(\frac{1}{m}\right)
$$

It is easy to see when $C_{2}$ is large enough, $\left\|s_{0}\right\|_{h_{0}}(z)$ can be as small as we want. Together we have

$$
\|s\|_{h}(0) \geq\|s\|_{h}(z)
$$

For case (iii), notice

$$
\begin{aligned}
e^{-m K}-e^{-m K_{m}} & =e^{-m K_{m}}\left(e^{-m\left(K-K_{m}\right)}-1\right) \\
m\left|K-K_{m}\right| & =-\sum_{i}\left(\frac{G^{0, \overline{e_{i}}}}{G^{0, \overline{0}}} z^{i}+\frac{G^{e_{i}, \overline{0}}}{G^{0,0}} \bar{z}^{i}\right)+O\left(|z|^{2}\right)
\end{aligned}
$$

Since $|z|^{2}<\frac{C_{2}}{m}$, we have

$$
m\left|K-K_{m}\right|=O\left(\frac{1}{m}\right)
$$

So in this case we have

$$
\|s\|_{h}-\|s\|_{h_{0}}=O\left(\frac{1}{m}\right)\|s\|_{h_{0}}
$$

For any $z$ such that $\frac{C_{1}}{m} \leq|z|^{2} \leq \frac{C_{2}}{m}$, we have

$$
\begin{aligned}
\|s\|_{h}(z) & =\left\|s_{0}\right\|_{h_{0}}(z)+\left(\|s\|_{h}(z)-\|s\|_{h_{0}}(z)\right)+\left(\|s\|_{h_{0}}(z)-\left\|s_{0}\right\|_{h_{0}}(z)\right) \\
& \leq\left\|s_{0}\right\|_{h_{0}}(z)+O\left(\frac{1}{m}\right)\|s\|_{h_{0}}(z)+\left\|s_{1}\right\|_{h_{0}}(z) \\
& \leq\left(1+O\left(\frac{1}{m}\right)\right)\left\|s_{0}\right\|_{h_{0}}(z)+\left\|s_{1}\right\|_{h_{0}}(z)
\end{aligned}
$$

It is easy to show that for $z$ in our range

$$
\left\|s_{0}\right\|_{h_{0}}(z) \leq C_{3}<1
$$


where $C_{3}$ depends on $C_{1}$. When $m$ is large enough, we have

$$
\|s\|_{h}(z) \leq\left(1+O\left(\frac{1}{m}\right)\right) C_{3}+\frac{1}{m}|z|<1=\|s\|_{h}(0)
$$

For case (ii), it is easy to show that

$$
\|s\|_{h}=1-C m|z|^{2}+O\left(m^{2}|z|^{4}+m|z|^{3}\right)
$$

which clearly achieves its maximum at $z=0$ for $|z|^{2} \leq \frac{C_{1}}{m}$ with $C_{1}$ small enough.

Now all the three cases are proved.

The case $L$ is only ample is a simple corrollary of the case when $L$ is very ample. One way to see this is that we can find two big primes $p_{1}, p_{2}$, such that $L^{\otimes p_{1}}$ and $L^{\otimes p_{2}}$ are very ample. From elementary number theory, we have that there exists an integer $N$, such that when $n \geq N$, we have

$$
n=a p_{1}+b p_{2}, \text { for some } a, b \geq n_{0} .
$$

Here the $n_{0}$ is the $n_{0}$ for the very ample case. Now it is obvious that $L^{\otimes n}=\left(L^{\otimes p_{1}}\right)^{\otimes a} \otimes\left(L^{\otimes p_{2}}\right)^{\otimes b}$ will have the sections we need.

Acknowledgment. I would like to thank Prof. M. Kontsevich for very stimulating discussion on mirror symmetry and Prof. Shouwu Zhang for proposing the problem in the last section. I also thank Prof. S. T. Yau, Prof. M. Weber and Prof. K. Koehler for pointing out the references leading to [B], Prof. Gang Tian for interest in my work. The work is done while my stay in IHES of France and Ruhr University at Bochum of Germany. I would like to thank both institutes for hospitality.

\section{References.}

[B] S. Bochner, Curvature in Hermitian metric, Bull. Amer. Math. Soc. 53 (1947), 179-195.

[H] L. Hörmander, An introduction to complex analysis in several variables, Van Nostrand, Princeton NJ, 1973.

[T] G. Tian, On a set of polarized Kähler metrics on algebraic manifolds, J. Differential Geometry, 32 (1990), 99-130. 
[Y] S-T. Yau, Nonlinear analysis in geometry, Enseignement Math. 33 (1986), 109-156.

RECEIVED OCTOBER 28, 1996.

DEPARTMENT OF MATHEMATICS

COLUMBIA UNIVERSITY

NEW YORK, NY 10027

E-MAIL: RUAN@SHIRE.MATH.COLUMBIA.EDU 